\title{
Statistical study of the influence of CNTs purification and plasma functionalization on the properties of Polycarbonate-CNTs nanocomposites
}

\begin{tabular}{|r|l|}
\hline Journal: & Plasma Processes and Polymers \\
\hline Manuscript ID: & ppap.201400008.R2 \\
\hline Wiley - Manuscript type: & Full Paper \\
\hline Date Submitted by the Author: & n/a \\
\hline Complete List of Authors: & $\begin{array}{l}\text { Maio, Andrea; University of Palermo, Dipartimento di Ingegneria Civile, } \\
\text { Ambientale, Aerospaziale, dei Materiali } \\
\text { Botta, Luigi; University of Palermo, Dipartimento di Ingegneria Civile, } \\
\text { Ambientale, Aerospaziale, dei Materiali } \\
\text { Tito, Alina Carmen; Polytechnic University of Milan, Department of } \\
\text { Chemistry, Materials and Chemical Engineering "Giulio Natta" } \\
\text { Pellegrino, Lorenzo; Polytechnic University of Milan, Department of } \\
\text { Chemistry, Materials and Chemical Engineering "Giulio Natta" } \\
\text { Daghetta, Matteo; Polytechnic University of Milan, Department of } \\
\text { Chemistry, Materials and Chemical Engineering "Giulio Natta" } \\
\text { Scaffaro, Roberto; University of Palermo, Dipartimento di Ingegneria Civile, } \\
\text { Ambientale, Aerospaziale, dei Materiali }\end{array}$ \\
\hline \multirow{2}{*}{ Keywords: } & \begin{tabular}{l} 
\\
\hline
\end{tabular} \\
\hline
\end{tabular}

\section{SCHOLARONE ${ }^{\text {Tw }}$}


((DOI: 10.1002/ppap.201100001))

Article type: full paper

\title{
Statistical study of the influence of CNTs purification and plasma functionalization on the properties of Polycarbonate-CNTs nanocomposites. ${ }^{a}$
}

\author{
Andrea Maio, Luigi Botta, Alina Carmen Tito, Lorenzo Pellegrino, Matteo Daghetta, Roberto \\ Scaffaro*
}

Dr. A. Maio, Dr. L. Botta, Prof. R. Scaffaro

Department of civil, Environmental, Aerospace, Materials Engineering, University of Palermo viale delle Scienze, Ed. 6, 90128 Palermo, Italy

E-mail: roberto.scaffaro@unipa.it

A. C. Tito, L. Pellegrino, Dr. M. Daghetta

Department of Chemistry, Materials and Chemical Engineering "Giulio Natta", Politecnico di Milano, Piazza Leonardo da Vinci, 32, 20133 Milan, Italy

\begin{abstract}
This work reports a statistical study on the relationship between the chemical-physical properties of CNTs, which vary by changing the conditions of purification and plasma treatment, and the macroscopic properties of PC-based nanocomposites. CNTs are synthesized and then purified in two different ways and used as fillers for the preparation of $\mathrm{PC} / \mathrm{CNTs}$ nanocomposites. In some cases, oxygen plasma treatment is carried out to improve their affinity to the matrix. The CNTs are characterized by TGA, ICP/OES and FT-Raman spectroscopy, titration and morphological analysis. Mechanical, dynamic-mechanical, electrical and morphological tests are used for characterizing PC/CNTs nanocomposites.
\end{abstract}

\footnotetext{
${ }^{\text {a }}$ Supporting Information is available at Wiley Online Library or from the author. 


\section{Introduction}

Since their discovery, carbon nanotubes (CNTs) attracted great interest, due to their excellent electrical, mechanical and thermal properties. ${ }^{[1-5]}$ The nanometric size and the structure, consisting of only carbon-carbon covalent bonds, make them one of the most interesting materials in nanotechnology field. ${ }^{[1-5]}$ CNTs main applications include the use as field emitters, ${ }^{[1]}$ nanoprobes and sensors, ${ }^{[2-5]}$ fillers for polymeric, ceramic and metallic composites. ${ }^{[6-34]}$ For some of these potential applications, as-prepared CNTs need to be purified, removing carbonaceous by-products (amorphous carbon, graphene, fullerenes) and catalyst residues. Among the application fields of CNTs, many attentions are devoted to their use as fillers to produce polymer based nanocomposites. In this perspective, the system polycarbonate/CNTs is one of the most investigated because of the possibility to combine the mechanical properties of the polycarbonate (PC) with the extraordinary mechanical and electrical properties of CNTs. ${ }^{[13-15]}$

Among the numerous preparation paths, CNTs prepared by Fluidized Bed Catalytic Chemical Vapour Deposition (FBCCVD) offers several advantages since it allows obtaining CNTs almost rid of carbonaceous by-products, the main impurities consisting either in catalyst particles and support grains wrapped by CNTs tangles or by catalyst nanoparticles embedded in the nanotubes. ${ }^{[12,35-42]}$ As-synthesized CNTs need to be purified, in order to remove the catalyst residue, which is undesired for most applications, since the presence of transition metals is commonly harmful for safety and health reasons. ${ }^{[43]}$ Moreover, depending on the final product, metal impurities could dramatically affect properties such as flame retardancy and polymer degradation/stability. Indeed it was well shown that iron impurities of as-prepared CNTs have a catalytic role in both polymer degradation and CNTs combustion, avoiding the material to resist under flame conditions. ${ }^{[44-46]}$

In order to promote the adhesion with a specific polymeric matrix, CNTs are often modified/functionalized using different methods. ${ }^{[13-15,18-20]}$ From an industrial point of view, 
the most interesting methods are those operating in solventless environments like plasma functionalization. This method was already successfully adopted to obtain polyamide $6 /$ CNTs nanocomposites with enhanced properties. ${ }^{[16-18]}$ The results demonstrated that it was possible to impart oxygen moieties on the sidewalls of CNTs without disrupting the structure of nanotubes. Moreover, being the plasma functionalization a completely solvent-free process, it has a low toxicity beyond being rapid and versatile.

In this work, multi-walled CNTs were synthesized by FBCCVD with the aim to study the relationships between the CNTs synthesis/purification conditions, the eventual plasma treatment and the macroscopic properties of PC/CNTs nanocomposites prepared by melt processing. Two typologies of CNTs with different characteristics (such as L/D and purity) were added to PC and the nanocomposites prepared in the melt have been investigated from a mechanical, thermo-mechanical and electrical point of view, using a two level full factorial statistical design. More in particular, different CNTs purity, plasma-functionalization and different filler content were applied in order to find out if the change of these parameters causes statistically significant variations of tensile elastic modulus, flexural storage modulus and electrical conductivity of the materials.

\section{Experimental Section}

\section{CNTs production: synthesis, purification, plasma functionalization}

Pristine CNTs were synthesized by fluidized bed chemical vapour deposition in a quartz reactor. ${ }^{[12]}$ The process uses a $\gamma$-alumina substrate impregnated with a catalyst (iron) as bed material. The iron content was $10 \mathrm{wt} \%$ or $20 \mathrm{wt} \%$. Ethylene was used as carbon source and nitrogen as fluidizing agent. The CNTs production yield of the process was $500 \mathrm{~g} / \mathrm{h}$ and the 
catalyst showed a nanotubes selectivity close to $100 \%$, as no amorphous carbon was found in the final product.

The purification process consists in three steps: i) acid treatment, ii) washing and iii) drying. CNTs batch size 50-300 g can be treated by this equipment, i.e. a quantity an order of magnitude higher than that reported in the scientific literature. ${ }^{[18-20,35-39]}$ High values of final purity and reproducible results can be achieved in relatively short times (150 minutes) and at relatively low temperatures $\left(120{ }^{\circ} \mathrm{C}-130{ }^{\circ} \mathrm{C}\right)$. Evaporative crystallization of waste waters allows recovering aluminium and iron sulphates to be recycled for catalyst preparation.

CNTs synthesized with a $20 \mathrm{wt} \%$ Fe catalyst may have a catalyst residue ranging from $13.05 \%$ to $33.21 \%$ (respectively $6.85 \mathrm{wt} \%$ of aluminium, $3.5 \mathrm{wt} \%$ of iron, $2.7 \%$ of oxygen, and $11 \mathrm{wt} \%$ of aluminium, $4.4 \mathrm{wt} \%$ of iron, $17.81 \%$ of oxygen) within the same nanotubes production batch. Further details about the synthesis process and the reactor are available in our previous work. ${ }^{[18]}$ A treatment with $45 \mathrm{wt}^{\%} \mathrm{H}_{2} \mathrm{SO}_{4}$ solution for 150 minutes allows achieving a final nanotubes purity ranging from $91.67 \%$ to $96.52 \%$. It should be outlined that, as the concentration of acid solution increases, the volumes of water required for washing increase too (from $15 \mathrm{~L}$ with $45 \mathrm{wt} \%$ solution to $27 \mathrm{~L}$ with $70 \mathrm{wt} \% \mathrm{H}_{2} \mathrm{SO}_{4}$ ).

The acid treatment removes almost completely the catalyst support (aluminium oxide), whereas iron is left in small percentage $(<1 \%)$ as a result of catalyst particles encapsulation at CNTs tips by the carbon shell and it selectively acts on the catalyst.

All the three process steps (acid reflux, washing, drying) are carried out in the same device: a three-phase slurry bubble column in which a nitrogen stream ensures bed motion. In order to assess the possibility to reduce the volume of water required for the washing, the CNTs purified with 45 wt $\% \mathrm{H}_{2} \mathrm{SO}_{4}$ solution for 150 minutes bearing a purity of $96.52 \%, \mathrm{~L} / \mathrm{D}=670$ 700, named CNT 1 were compared to CNTs purified with $70 \%$ wt $\mathrm{H}_{2} \mathrm{SO}_{4}$ solution for 150 minutes (CNT 2), characterized by purity degree of $99.1 \%$ and a L/D ratio equal to $890-900$. 
Both samples underwent a surface oxidation by oxygen-plasma treatment. The plasma reactor used for the surface treatment is a Tucano Gambetti apparatus, showed in Scheme 1, equipped with a high vacuum-pump (Pfeiffer). The cylindrical chamber (diameter $=150 \mathrm{~mm}$, length $=330 \mathrm{~mm}$ ) has a volume of approximately 5.5 litres. The dark shield electrode and the power supply (RF $13.56 \mathrm{MHz}, 200 \mathrm{~W}$ ) are placed at the top of the chamber. The flow of the oxygen takes place automatically at vacuum level and power programmed in the recipes with two mass flow-controllers, and it is controlled by a microcomputer.

In this case, the oxygen flow was set to $7 \mathrm{sccm}$, the vacuum level was $210^{-4}$ bar of pure oxygen; it was achieved after 3 refluxes of pure oxygen to eliminate the air residual and the vacuum was stabilized for 5 seconds prior to the process gas inlet. After gas inlet the pressure in the chamber reached a value of about 0.5 mbar and the treatment has been then carried out at $120 \mathrm{~W}$ for 10 minutes. The parameters were adjusted in order to minimize reverse bias. Other details about the choice of these parameters can be found in our previous works. ${ }^{[16-18]}$

\section{CNTs characterization}

Purified CNTs were characterized using High Resolution Transmission Electron Microscopy (HRTEM, JEOL, JEM-2100 LaB 6 ) and Scanning Electron Microscopy (SEM, ZEISS EVO ${ }^{\circledR} 50 E P$ ), providing a qualitative analysis of CNTs samples; particles size analysis (CILAS $1180 \mathrm{~L})$ and HRTEM were used to show respectively size and structural differences between as-synthesized and purified CNTs, Thermogravimetric Analysis-Differential Thermogravimetry (TGA-DTG, TA SDT Q600) and inductively coupled plasma optical emission spectrometry (ICP/OES, Perkin Elmer Optima 200DV) were used to quantify CNTs purity, their composition and hence treatment efficiency. X-ray diffraction analysis (Bruker $A X S-D 8$ Advance using CuK $\alpha$ radiation, in the $2 \theta$ range $20-30^{\circ}$ ) was used to define better the composition of residual catalyst (obtained by an air oxidative treatment of nanotubes). 
Fourier Transformed Raman spectroscopy (BRUKER, RFS 100/S) using Nd:YAG laser $(1064 \mathrm{~nm})$ was used to confirm the graphitized carbon nanotubes structure and to investigate qualitatively the degree of purification.

A typical acid-base titration was used to determine the surface functional groups. The oxidized CNTs $(100 \mathrm{mg})$ have been added to a $\mathrm{NaOH}$ aqueous solution $(25 \mathrm{~mL}-0.050 \mathrm{~N})$ and the resulting suspension has been stirred for 48 hours at room temperature in a closed vessel. The solid was then filtered and $5 \mathrm{~mL}$ aliquot of the solution was titrated with $\mathrm{HCl}(5.4 \mathrm{~mL}-$ $0.046 \mathrm{~N})$. The $\mathrm{pH}$ variation was monitored using a pHmeter (827 pHLab, Metrohm). The difference between the initial $\mathrm{OH}^{-}$moles and the titrated ones corresponds to the moles of reacted $\mathrm{COOH}$ groups, which resulted $389 \mu \mathrm{mol}$ per gram of nanotubes.

\section{Preparation and characterization of polymer based nanocomposites}

The PC used in the frame of this work was a sample of Lexan HF 1110, which main characteristics are summarized in Table $\mathbf{1 .}$

Two kinds of fillers were used both as synthesized/purified and functionalized at two different loading levels $(0.5 \%$ and $1 \%)$ to investigate the effect of concentration, functionalization and typology of the CNTs on the properties of PC-based nanocomposites which were obtained by melt processing. The PC and CNTs, premixed at the solid state, were vacuum-dried at $140{ }^{\circ} \mathrm{C}$ overnight prior to using, and then fed to the mixer at $20 \mathrm{rpm}$ in no longer than $2 \mathrm{~min}$. Subsequently, the speed was increased up to $64 \mathrm{rpm}$ until a constant value of the mixing torque was achieved (usually after about 3 minutes). The temperature was kept constant at $280{ }^{\circ} \mathrm{C}$ all over the processing operation. Once mixing was completed, the material was fed out the mixer within 2 minutes, grinded and compression-moulded in a Carver (USA) laboratory press $\left(280^{\circ} \mathrm{C}, 100 \mathrm{bar}\right)$, cooled down and finally cut into specimens of appropriate dimensions for the further characterizations. 
Mechanical characterization was carried out on rectangular-shaped specimens cut off from compression-moulded sheets $(10 \times 90 \times \sim 0.6 \mathrm{~mm})$. Mechanical testing was performed using an Instron 3365 universal tensile testing machine according to ASTM D882 on at least 10 replicates. The grip distance was set to $30 \mathrm{~mm}$ and the crosshead speed was $5 \mathrm{~mm} / \mathrm{min}$. The elastic modulus was calculated as the slope of each stress-strain curve extrapolated at zerostrain.

Thermo-dynamic-mechanical analysis was carried out by using a DMTA V Rheometrics Scientific equipment. The PC based nanocomposites were tested in the single cantilever flexural mode. The specimen, $4 \mathrm{~mm}$ thick, $35 \mathrm{~mm}$ long and $8 \mathrm{~mm}$ wide, was clamped on the testing head and stressed with a small tensile pre-charge. Then it was inserted in an insulated thermostatic chamber under nitrogen flow. The test was carried out in the temperature sweep mode in the range $50{ }^{\circ} \mathrm{C}$ - $200{ }^{\circ} \mathrm{C}$ at a heating speed of $5{ }^{\circ} \mathrm{C} / \mathrm{min}$. The maximum strain amplitude applied was $0.1 \%$ while the testing frequency was $1 \mathrm{~Hz}(6.28 \mathrm{rad} / \mathrm{s})$.

Electrical resistance of the samples was measured using a four probe cell and a Keithley 2612 multimeter. The data were further used to calculate the electrical conductivity of the materials by the first and the second Ohm's law.

The morphological analysis (SEM and TEM) was carried out in the same apparatuses used for the CNTs but different preparative are adopted. For SEM analysis, all samples were fractured in liquid nitrogen and sputter-coated with a thin layer of gold to avoid electrostatic charging under the electron beam. As regards the TEM analysis, the samples of nanocomposites were cut into slices of $150 \mathrm{~nm}$ thick using an ultra-cryo-microtome and placed on a lacey carbon grid (with a core made in copper) and analyzed by a JEOL 2100 operating at $200 \mathrm{KV}$. 


\section{Methods}

All the data were statistically analyzed by a two level full factorial design. This method, better discussed in our previous work ${ }^{[47]}$ and in the SI, allows assessing the main effect of process parameters and the effect of binary interactions between the variables within the range investigated.

Synthetically, a generic property $\mathrm{P}\left(x_{1}, x_{2}, \ldots, x_{n}\right)$ can be investigated and described by choosing two-levels for each of the $\left(x_{1}, x_{2}, \ldots, x_{n}\right)$ variables, thus obtaining $2^{\mathrm{n}}$ combinations to be taken into account in the experimental analysis. These combinations are usually schematically summarized in a "table of contrasts" (see SI), where each row characterizes a specific conditions set, while the columns allow calculating the main effects of each variable and the interactions between the variables, being the main effect of a variable the difference of two averages (one related to the superior level of the variable, the other related to the inferior level). Therefore, in this way it is possible to appreciate the influence of each variable on the selected property, keeping the other variables constant; e.g. the main effect of the $x_{1}$ variable, $\mathrm{Px}_{1}$, provides the increment that the $\mathrm{P}$ property gets when varying between the inferior and the superior level of the variable. In the statistical calculations, the table of contrasts is filled with "+" and "-" signs, depending on the considered variable assuming its superior or inferior level, respectively. The last column reports the numerical values that the $\mathrm{P}$ property assumes for each of the $2^{n}$ sets of conditions. It can be demonstrated that the main effect of the generic " $i$ " variable can be calculated as the algebraic sum of the values of $P$, each taken with the sign which appears in the corresponding column of the table.

Of course, a detailed design cannot be based on the sole determination of main effects, which provide an incomplete description of the influence exerted by each variable on the selected property, but requires assessing also the significance of the possible interactions between each 
variable. In the table of contrasts, the column of the interaction between the variables $x_{1}$ and $x_{2}$ is obtained by multiplying the sign arrays of the two columns of variables.

In this work, the properties investigated were Elastic modulus outcome from tensile tests, flexural storage modulus measured at $50{ }^{\circ} \mathrm{C}$ by DMTA and electrical conductivity derived by electrical measurements. The number of variables was 3:

1) effect of filler concentration; inferior level (-): $0.5 \% \mathrm{wt} / \mathrm{wt}$; superior level $(+): 1 \%$;

2) effect of functionalization (inferior level: pristine CNTs, superior level: plasmatreated by oxygen at $120 \mathrm{~W}$ for 10 mins);

3) effect of typology of CNTs (inferior level: purified by a $55 \%$ wt $\mathrm{H}_{2} \mathrm{SO}_{4}$ solution for 150 mins, superior level: purified by a $70 \%$ wt $\mathrm{H}_{2} \mathrm{SO}_{4}$ solution for $150 \mathrm{mins}$ ).

The binary interactions investigated were, of course, those between filler concentration and functionalization (1-2), filler concentration and typology of filler (1-3), functionalization and typology of filler (2-3).

Moreover, the analysis of variance (ANOVA) was applied to the full factorial design above described, since it is a powerful tool to verify which variable change has a statistically significant influence. Again, more details are included in the supporting information, however it is useful to give a brief description of the method.

For the eight materials obtained with all the possible combination of the two levels of the three variables $\left(2^{3}\right.$ experiments), we took into account the value range (min-max) for each sample, the square of the difference between the maximum and the minimum value for each sample $\left(d_{i}^{2}\right)$ and $d_{i}^{2} / 2 g$ where $g$ is the number of the degree of freedom (in our case $2^{3}=8$ ).

It is then possible to calculate the estimated experimental run variance $s^{2}$ as follows:

$s^{2}=\sum\left(s i^{2} / g\right)=\sum\left(d i^{2} / g\right)$

and then the mean variance $\mathrm{V}(\mathrm{E})$ as: 
$\mathrm{VE}=(1 / g+1 / g) s^{2}$

and its standard error SE calculated as the square root of $\mathrm{V}(\mathrm{E})$.

For the minimum threshold of statistical significance (MTSS), instead, the method which has been adopted in this work is based on the consideration that each ratio, effect/SE (effect), will be distributed in a $t$ distribution with $g$ degrees of freedom. Therefore, choosing a proper confidence level (here, 95\%) the execution of a $t$-test allows assessing the statistical significance of each effect (and thus of its related variable, or between-variable interaction) at that confidence level.

The change of a certain property due to the change of a certain parameter has a statistically significant difference if the related main effect is higher than MTSS.

\section{Results and Discussion}

In order to optimize the purification conditions of the CNTs, the degree of purity and the composition of impurities were studied as a function of time and $\mathrm{H}_{2} \mathrm{SO}_{4}$ concentration of the solution. In particular, the degree of purity was calculated by measuring the residue after TGA, reported in Figure 1 a-b. The composition of the impurities was calculated by performing ICP-OES spectroscopy on the residue outcome from TGA in order to assess the amount of Fe and Al. The wavelengths used for the detection of Fe were 238.205, 239.571 and $259.940 \mathrm{~nm}$; for the detection of $\mathrm{Al}$, instead, the wavelengths used were: 396.158 , 308.218 and $394.410 \mathrm{~nm}$, different from those about iron in order to avoid interference in the spectra. Table 2 summarizes the ICP/OES data(i.e. the amounts of metal residues) and the degree of purity (TGA data) over time for CNTs purified adopting different acid concentrations. The graphic interpretation of these data is showed in Figure $\mathbf{2} \mathbf{a}-\mathbf{b}$, where the residual weight of catalyst, $\mathrm{Fe}$ and $\mathrm{Al}$ is plotted as a function of the purification time for the system acid-treated with respectively $45 \mathrm{wt} \%$ (a) and $70 \mathrm{wt} \%$ (b) $\mathrm{H}_{2} \mathrm{SO}_{4}$. 
It is evident that by using more concentrated solutions (70 $\mathrm{wt} \%)$, a plateau is reached after only one hour while with the lower acid concentration the purity monotonically increases up to the purification times. ICP/EOS results show that aluminium is completely removed after 30 minutes using the $70 \mathrm{wt} \% \mathrm{H}_{2} \mathrm{SO}_{4}$ solution. On the contrary, iron residue shows a different trend: for all the tested acid concentrations, it settles around values of one percentage point and it does not reach values below $0.7 \%$ even with $70 \mathrm{wt} \%$ acid solution.

The TEM images reported in Figure 3 show the as-synthesized CNTs, Figure 3 a-b, the CNTs purified with $45 \mathrm{wt} \% \mathrm{H}_{2} \mathrm{SO}_{4}$ acid for 150 minutes, Figure 3 c-d, and the CNTs purified with a $70 \mathrm{wt} \% \mathrm{H}_{2} \mathrm{SO}_{4}$ solution for 150 minutes, Figure 3 e-f. The micrographs clearly indicate that the CNTs are heterogeneous both before and after purification treatments and many defects are observed, such as opening ends, bending, cutting (outlined by circles inside the figures) and amorphous carbon formation. As the oxidative treatment can cause structural damages in the sample, it is possible to quantify the amounts of defects by Raman analysis. In In particular, this method allows taking into account that these defects induce disorder into the CNTs graphitic structure breaking the regularity of the nanotubes,. For this purpose, FTRaman spectra have been collected for all the samples and reported in Figure 4. All the spectra show two peaks at $1365 \mathrm{~cm}^{-1}$ and $1583 \mathrm{~cm}^{-1}$. The peak at $1583 \mathrm{~cm}^{-1}$ (G band) corresponds to the Raman allowed optical mode $\mathrm{E}_{2 \mathrm{~g}}$ of two-dimensional graphite, while the peak at $1365 \mathrm{~cm}^{-1}$ (D band) is mainly derived from disordered carbon defects of the CNTs, from amorphous carbon and finite size of tubes crystalline domains. ${ }^{[48]}$ After the acid treatment, these two bands can still be found, indicating that the whole structure of nanotubes is not destroyed.

It was found that the $\mathrm{I}_{\mathrm{D}} / \mathrm{I}_{\mathrm{G}}$ intensity ratio measurable by FT-Raman can give anidea on the disorder amount. ${ }^{[16-18]}$ In Table 3 there are reported the $\mathrm{I}_{\mathrm{D}} / \mathrm{I}_{\mathrm{G}}$ values of the samples derived from Figure 4. It can be noted that the ratio slightly increased after the purification process. 
As commonly accepted, treatments with oxidizing agents like nitric acid could introduce functional groups on CNTs surface. ${ }^{[49-53]}$ In particular, it is possible to find hydroxyl and carboxyl groups, which are fundamental to improve, for instance, the adhesion between the nanofillers and the matrix in nanocomposite materials. ${ }^{[54-57]}$

In order to evaluate if an analogous oxidative effect can be performed by sulphuric acid, a direct acid-base titration technique using $\mathrm{NaOH}$ was performed to determine the concentration of surface groups. A sample of purified CNTs (with $70 \mathrm{wt} \%$ sulphuric acid solution for 150 minutes) was treated with a $\mathrm{NaOH}$ aqueous solution for 48 hours. The unreacted hydroxide has been then titrated with $\mathrm{HCl}$, following the reaction with a pHmeter. The reacted amount of acid groups was deduced and evaluated in terms of millimoles of $\mathrm{COOH}$ per grams of CNTs. The treatment with sulphuric acid does not induce an high functionalization degree in the samples (average $\mathrm{mmol} \mathrm{COOH} / \mathrm{g}=0.389$ ), in agreement with the defects level observed by TEM and with the increase of the $\mathrm{I}_{\mathrm{D}} / \mathrm{I}_{\mathrm{G}}$ ratio from Raman spectra that is therefore validated as a rapid, although qualitative, method to evaluate the functionalization degree of the nanotubes. Anyhow, in order to impart a larger amount of oxygenated moieties, plasma treatment $(10$ minutes and $120 \mathrm{~W})$ was carried out on the two samples of CNTs. In the same Table 3 there are reported the $\mathrm{I}_{\mathrm{D}} / \mathrm{I}_{\mathrm{G}}$ ratios of functionalized CNTs (f-CNT1 and $\mathrm{f}-\mathrm{CNT} 2$ ). The $\mathrm{I}_{\mathrm{D}} / \mathrm{I}_{\mathrm{G}}$ ratios indicate that $\mathrm{f}-\mathrm{CNT}$ treated show a higher disorder degree if compared to the untreated CNTs, suggesting a higher oxidation level. In particular, the functionalization of $\mathrm{CNT} 2$ at $120 \mathrm{~W}$ for 10 minutes gives rise to the highest $\mathrm{I}_{\mathrm{D}} / \mathrm{I}_{\mathrm{G}}$ ratio suggesting the highest functionalization level.

All the CNTs samples prepared and characterized as discussed above were melt blended with polycarbonate and characterized from a mechanical and electrical point of view. The results were interpreted by using a two level Full Factorial analysis. In detail, the referring parameters were the filler concentration, the typology of nanotubes i.e. the different purification level, the presence or not of plasma functionalization. The aim of this analysis is 
to find a quantitative correlation between these variables to find the set of parameters to be used to have the best performance of the final material.

In particular, the analysis of variance (ANOVA) applied to the full factorial design above described, is a powerful tool to verify which variable change has a statistically significant influence. More details are included in the supporting information, however it is useful to give a brief description of the method.

In Table 4 it is reported the Matrix of the contrasts, in which the 8 different operative sets $\left(2^{3}\right.$, as already discussed in "Methods") are indicated with the letters from A to $\mathrm{H}$ in the first column. The second, third and fourth columns report in details the level used for the three variables, i.e. CNTs concentration (1), functionalization (2) and CNTs typology (3). The columns from $5^{\text {th }}$ to $7^{\text {th }}$ are, the columns of the binary interactions between the variables and they are obtained by multiplying the sign arrays of the two columns of variables. As already discussed, since " +" symbol represents the upper level of the specified variable, and the "_." symbol the lower one, the main effect of each variable (or interaction), is calculated according to the Eq. (3):

$$
\text { Main Effect }=\sum_{i=A}^{H} \mathrm{Mi}
$$

where $i=(A, B, \ldots, H)$ are the eight specified sets of conditions, Mi is the average value of the tensile modulus in each set of conditions (as reported in the $8^{\text {th }}$ column of the matrix of the contrasts) and the sum is calculated taking the generic "Mi" value as positive or negative depending on the sign ( "+" or "-") it assumes in the assigned column of the table of contrasts. The last two columns $\left(9^{\text {th }}\right.$ and $\left.10^{\text {th }}\right)$ report the elastic modulus of nanocomposites upon the elastic modulus of the pure matrix $\left(\mathrm{E}_{\mathrm{m}}\right)$ and the percentage variation with respect to pure matrix, defined as: 


$$
\frac{E-E m}{E} \times 100
$$

The 8 experimental runs gives results with a certain data scattering, as illustrated in the charts reported in Figure 5, which was interpreted and evaluated by ANOVA.

In Table 5 it is reported the ANOVA analysis applied to the tensile modulus. In the first column there are the codes of the eight materials obtained with all the possible combination of the two levels of the three variables $\left(2^{3}\right.$ experiments). The following columns are the value range (min-max) for each sample, the square of the difference between the maximum and the minimum value for each sample $\left(\mathrm{d}_{\mathrm{i}}^{2}\right)$ and $\mathrm{d}_{\mathrm{i}}^{2} / 2 \mathrm{~g}$ where $\mathrm{g}$ is the number of the degree of freedom (in our case $2^{3}=8$ ).

The estimated experimental run variance $s^{2}$ was calculated by eq. (1) while the mean variance, $\mathrm{V}(\mathrm{E})$, is determined according to eq. (2)

and its standard error SE was calculated as the square root of $\mathrm{V}(\mathrm{E})$.

The execution of a $t$-test at $95 \%$ confidence level, stated that the change of a certain property due to the change of a certain parameter has a statistically significant difference if the related main effect is 2.3 times higher than SE; differently, the variations between the measured values should be considered mere changes within the experimental error. In the reported case, the minimum threshold of statistic significance, MTSS (=2.3 SE), is about $66 \mathrm{MPa}$.

The numerical data about the main effect of the three variables and the binary interactions are reported in the last row of the matrix of contrasts. By comparing them with the MTSS (66 $\mathrm{MPa}$ ) carried out from ANOVA, it is possible to state that all the variables have a strong influence on the tensile modulus (within the range investigated). In particular, this latter is increasing by increasing the filler concentration, by applying plasma functionalization and by using CNTs with a higher L/D ratio and a higher purity. The most influent variable is the CNTs type, followed by the plasma functionalization, while the filler concentration, has the lowest effect on this property. 
The graphical interpretation of the influence of the three variables is depicted in Figure 6. By observing the high slope of all the three lines associated with each single variable, it can be concluded that they all have a significant influence on the examined property, in this case the tensile modulus, thus confirming the data outcome from Table 4.

The interactions can be also studied by analyzing the matrix of contrasts and in particular by combining the three columns into other columns (as aforementioned) describing, each, the effect of the interaction between a couple of variables. Again, if the resulting absolute value of the main effect for a certain couple is significantly different from the minimum threshold of statistical significance (66 MPa) those two variables have a significant interaction on the final value of the property, differently they have not. This can be also reported in graphs, as depicted in Figure 7 that can be considered the map of the binary interactions. The two lines (called Box-Hunter lines) may be incident in the investigated range, out of the investigated range or may be almost parallel.

When the lines are incident, before and after the intersection the behavior is dominated by either one or another variable, indicating an interaction between the two. This interaction is stronger when the slopes are more different.

The intersection can falls out the investigated range. In this case, the intersection may have a physical meaning or it can be completely meaningless (e.g. a negative volume). When it occurs, the interaction is considered from weak to absent. Same is the case of almost parallel lines.

In our case, the filler content-functionalization type, Figure 7a, does not show any significant interaction. In fact, the intersection is below $0 \%$ of filler, meaning that its decrease could cause a decrease of modulus by using O-f-CNT only at negative concentration i.e. without any significant physical meaning. The interaction between CNT type and CNT concentration, 
Figure $7 \mathbf{b}$, shows the highest slope difference but anyway the intersection falls close to $0 \%$ with the same consideration reported above. In this case, however, the different slopes (the two lines are far from being almost parallel) indicate that there is a synergism between the concentration and the CNT type: when using CNT1 the modulus is almost constant on increasing the concentration, while the CNT2 containing materials show a relevant increase. As regards the interactions between the power and the CNTs type, Figure 7c, the intersection falls at about $250 \mathrm{~W}$ power. Although this value has some physical significance, it is not in the range here investigated since, a value of power higher than $150 \mathrm{~W}$ could cause remarkable damage to the bulk structure of the nanotubes (i.e. strong ablation of the outer walls). ${ }^{[16]}$ It can be concluded that the interactions between the variables are substantially weak except for the filler content vs CNT type couple. These latter result opens to a discussion about the reason of this synergism. It can be hypothesized, in fact, that using CNT2 instead of CNT1 allows having a better dispersion and adhesion. with the PC matrix. In this case the stresses are better transmitted from the matrix to the filler and a higher value of the modulus is shown.

This hypothesis is confirmed by SEM analysis. In Figure 8 a-d, there are reported the SEM micrographs of PC nanocomposites with $1 \mathrm{wt} \%$ of CNT1, O-f-CNT1, CNT2 and O-f-CNT2. It is clearly seen, Figure 8a, that CNT1 nanotubes offer a poor interfacial adhesion with the matrix thus confirming the hypotheses above described. The situation is slightly better when CNT1 are plasma functionalized, Figure $\mathbf{8 b}$, as in this case some bridging phenomenon can be observed, as indicated by the circles on the micrograph. However, the best adhesion is observed when using CNT2, depicted in Figure 8c (CNT2) and Figure 8d (O-f-CNT2). The unfunctionalized CNT2 display a better adhesion with the PC matrix even if the dispersion is not excellent (some CNTs aggregates can be identified in the PC matrix) but when using O-fCNT both the dispersion and the adhesion are improved: although the presence of few pulled 
out nanotubes was still observed, even hybrid structures can be found, as highlighted in the micrograph.

A similar study was carried out on the flexural storage modulus measured at $50{ }^{\circ} \mathrm{C}$ during DMTA tests. The ANOVA, see Table S1 in the SI for more details, suggests that it is possible to attribute statistical significance only to the variables with main effects above $5.8 \cdot 10^{7}$. In Figure 9 it is plotted the main effect of content, typology and functionalization of the CNTs on the E' of PC/CNTs nanocomposites, obtained by the matrix of the contrasts, reported in Table S2. Similarly to the findings about the elastic modulus, also the flexural modulus is influenced by all the three variables.

The most influent variable is the typology of CNT followed by the plasma functionalization while the filler content has a low influence on this property: doubling the amount of CNTs the flexural modulus increase is limited. Nevertheless, the influence of CNT concentration has some statistical relevance (the main effect is about 10 times the experimental noise error) but its impact is sensibly lower if compared with the other two parameters. Moreover, the behavior of the elastic modulus as a function of the filler content in a larger range investigated is showed in Figure S1. The results put into evidence that this property does not follow a linear trend versus filler content, as already found in previous works. ${ }^{[14,16]}$ For all the CNTs used, the most relevant increment of the elastic modulus is observed when only $0.5 \mathrm{wt} \%$ by weight of CNTs is added. Above this level, the increase is less pronounced and the elastic modulus tends either to level off or to decrease above $1 \mathrm{wt} \%$ of filler content. Adding up to 1 wt $\%$ of CNTs, moreover, was found to cause a remarkable increase of the elastic modulus, due to the hindered mobility of the matrix polymeric chains within the composite and to the intrinsic reinforcing effect of the filler. When $2 \mathrm{wt} \%$ of CNTs are added their attitude to be dispersed within the matrix is reduced because of the oversaturation and, above all, because of the viscosity of the system during processing. This latter, in fact, increases on increasing the 
filler content and this feature causes a remarkable polymer matrix degradation. ${ }^{[14,56-60]}$ In order to confirm this hypothesis, the melt viscosity during processing was monitored by measuring the mixing torque. The final torque of the systems investigated was then plotted versus the filler content and reported in Figure S2. The nanocomposites filled with $1 \mathrm{wt} \%$ of CNTs exhibited final torque value ranging from 7.4 to $8.4 \mathrm{Nm}$, close enough to that observed for neat PC. Differently, the materials containing $2 \mathrm{wt} \%$ of CNTs showed values of about 10 $\mathrm{Nm}$ and, when f-CNT 2 are used, being the final torque $11 \mathrm{Nm}$, i.e. almost the double with respect to neat PC. The high influence of the CNT type on the flexural modulus was explained considering the different L/D ratio of the two sample of CNTs used in this work.

In fact, it known from the scientific literature that the flexural modulus is proportional to the $\mathrm{L} / \mathrm{D}$ ratio. ${ }^{[61]}$ On increasing the ratio between surface and volume $-\mathrm{A} / \mathrm{V}-$ of the filler, the interface surface increases too. When the interface is resistant i.e. strong enough the mechanical properties of the composite are better. In particular, if a is the L/D ratio, it can be found a correlation with $\mathrm{A} / \mathrm{V}$ ratio as those described by Eqn. 5: ${ }^{[61]}$

$\mathrm{A} / \mathrm{V}=(2 \pi / \mathrm{V})^{1 / 3}\left(\mathrm{a}^{-2 / 3}+\mathrm{a}^{1 / 3}\right)$

It can be easily found that the $\mathrm{A} / \mathrm{V}$ ratio is maximum for one-dimensional fillers $(\mathrm{a}>>1)$ and for lamellar fillers $(\mathrm{a}<<1)$. In our case CNT2 have values of $\mathrm{A} / \mathrm{V}$ between 800 and 900 , while CNT1 present a value of about 500. The values are somehow smaller for both samples after the functionalization as the plasma treatment causes a decrease of the CNTs diameter. However, while CNT1 after treatment show a value of below 700, CNT2 a value is about 900-1000 that induces higher mechanical properties i.e. in full agreement with the findings of the statistical analysis. 
As regards the binary interactions between the variables, it was found (see Figure $\mathbf{S 3}$ for more details) that there are no significant interactions and no synergistic or antagonist effects can be detected.

ANOVA was also used to study the statistically significant difference in the electrical conductivity of the samples and determined by comparing MTSS and the main effect. More details can be found in the SI, Table S3 and Table S4.

The graphical interpretation of the main effect of the three variables is reported in Figure 10. It can be seen that the only two significant variables are the concentration and the typology of the CNTs used while the functionalization is not apparently influencing the electrical conductivity i.e. the values obtained in the two conditions are not statistically different. The positive influence of CNT type and concentration, on the contrary, was expected as a higher level of CNTs increases the possibility to form a percolated CNTs path inside the material and/or to make more probable electron transfer by tunneling. It is also well known that electrical properties, particularly electrical conductivity, of CNT based nanocomposites show a percolation threshold, that was usually found to be $1 \%$ wt. of nanotube load. This is due to the formation of CNT networks inside the polymer matrix, which is crucial for electrical conductivity to appear. The formation of this network increases as the weight load of nanotubes increases, yielding an electrical conductivity improvement. Yet, it was also outlined that for concentrations of CNT above 4\% wt. no further improvement was observed, because CNTs tend to re-aggregate and the networks responsible of electrical conductivity tend to spread out. Moreover at higher CNT loadings, the morphology distribution of the filler, particularly its aspect ratio, could affect electrical conductivity, i.e. the presence of shorter CNTs was found not to yield a significant improvement of electrical behavior. ${ }^{[62]}$ On the other hand the higher quality CNTs (CNT2) offer a higher L/D ratio but also a higher purity, both favoring a better electrical conductivity. As regards the null effect of 
functionalization, it must be considered that the functionalization induce a better dispersion (that should increase the chances to form conductive paths) but it also partially destroy the CNTs structure and introduces oxygenated moieties that make CNTs less conductive. The results can be interpreted considering a sort of balance between the two effects i.e. the positive effect of the better dispersion is counterbalanced by their lower conductivity. Functionalization of carbon nanotubes usually occurs on tube surface, causing structural defects. Although the relative low abundance of defects on the nanotubes of the present work after acid treatment, more defects could be created after plasma functionalization, determining discontinuities in MWCNT bundles. Such discontinuities strongly affect electrical conductivity, as well outlined by several authors. ${ }^{[63-66]}$ In particular, it was observed that strongly functionalized MWCNT, e.g. with sulfonitric mixture, could have an electrical conductivity as lower as three orders of magnitude with respect to the untreated ones, thus suggesting that some types of wet chemistry reactions modify not only the surface but even the bulk. In this case, the CNTs were purified with a less aggressive acid solution and further functionalized with a plasma treatment that imparts structural changes that can be considered merely superficial. On the other hand, after functionalization, CNTs exhibited a better

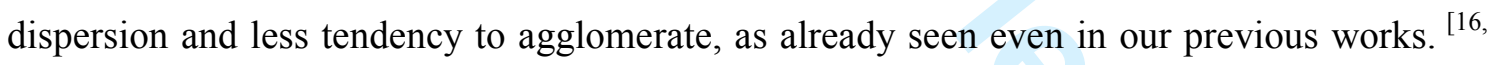
${ }^{18]}$ This feature was evaluated by performing TEM analysis, reported in Figure 11, directly onto the samples which underwent electrical test. It is clear as CNT2 are more aggregated than f-CNT2, thus confirming the hypothesis above described.

Moreover, the study of the binary interactions, reported in Figure S4, put into evidence a strong interaction between filler content and CNTs typology. This phenomenon can be explained by considering that CNT 2 show less tendency to form aggregates even at higher loading. 


\section{Conclusion}

Plasma treated CNTs allow improving mechanical and dynamic-mechanical properties of $\mathrm{PC} / \mathrm{CNTs}$ nanocomposites without affecting electrical properties. Based on statistical analysis performed on the nanocomposites, it was possible to evaluate that plasma-treated CNTs with lower aspect ratio and lower purity (CNT 1), give rise to nanocomposites with mechanical and dynamic-properties comparable to those showed by nanocomposites loaded with more purified CNTs (CNT 2). As plasma treatment is faster and simpler than analogue reactions performed in solution, it is possible to save time and solvent to obtain fillers that are able to improve the mechanical properties. Another relevant issue regards the electrical properties of these nanocomposites. Usually, functionalization of CNTs (especially by wet chemistry method) causes a remarkable decrease of electrical conductivity, while in the case of plasmafunctionalization (which occurs only in the outer walls of these nanoparticles) this phenomenon did not occur and, presumably due to the better dispersion of CNTs within the polymer matrix, the electrical properties remained almost constant and influenced only by CNTs concentration, as expected.

Received: ((will be filled in by the editorial staff)); Revised: ((will be filled in by the editorial staff)); Published online: DOI: 10.1002/ppap.201100001

Keywords: cold plasma; hydrophilic coatings; nanocomposites; optical emission spectroscopy (OES); full factorial design 
[1] W.I. Milne, K.B.K. Teo, G.A.J. Amaratunga, P. Legagneux, L. Gangloff, J.P. Schnell, V. Semet, V.T. Binh, O. Groening, J. Of Mater. Chem. 2004, 14, 1.

[2] J.M. Bonard, H. Kind, T. Stöckli, L.O. Nilsson, Solid-State Electronics 2001, 45, 893.

[3] H.J. Dai, J.H. Hafner, A.G. Rinzler, D.T. Colbert, R.E. Smalley, Nature, 1996, 384147.

[4] T. An, W. Choi, E. Lee, I. Kim, W. Moon, G. Lim, Nanoscale Res Lett. 2011, 6, 306.

[5] C. Li, E. T. Thostenson, T.W. Chou, Compos. Sci. Technol. 2008, 68, 1227

[6] E. T. Thostenson, Z. Ren, T.W. Chou, Compos. Sci. Technol. 2001, 61, 1899

[7] A. Agarwal, S.R. Bakshi, D. Lahiri, Carbon nanotubes: reinforced metal matrix composites ; Boca Raton, CRC Press, 2011, 305 pp.

[8] A. Rahman, I. Ali, S.M. Al Zahrani, R.H. Eleithy, NANO: Brief Rep. and Rev., 2011, 6(3), 185

[9] L. Bokobza, Polymer, 2007, 48(17), 4907

[10] R.Z. Ma, J. Wu, B.Q. Wei, J. Linag, D.H. Wu, J. Mater. Sc., 1998, 33, 5243

[11] G.D. Zhan, J.D. Kuntz, J.L. Wan, A.K. Mukherjee, Nature Mater., 2003, 2, 38

[12] J. Liu, O. M. Dunens, K. J. Mackenzie, C.H. See, A.T. Harris, AIChE Journal, 2008, 54(12), 3303

[13] P. Potschke, A. R. Bhattacharyya, A. Janke, Eur Polym J, 2004, 40, 137

[14] P. Potschke, A.R. Bhattacharyya, A. Janke, Composite interfaces, 2003, 10 (4-5), 389 
[15] U. A. Handge, P. Pötschke, Rheol Acta, 2007, 46, 889

[16] R. Scaffaro, A. Maio, S.P. Agnello, A. Glisenti, Plasma Process. Polym. 2012, 9, 503

[17] R. Scaffaro, A. Maio, Polym. Test. 2012, 31, 889

[18] R. Scaffaro, A. Maio, A.C. Tito, Compos. Sci. Tech. 2012, 72(15), 1918

[19] J. Liu, A.T. Harris, Chem. Engin. Sc., 2009, 64, 1511

[20] D. Golberg, Surface Modification of Nanotube Fillers; Vikas Mittal, 2011, 331 pp.

[21] H. Gu, J. Guo, Q. He, Y. Jiang, Y. Huang, N. Haldolaarachige, Z. Luo, D.P. Young, S.Wei, Z. Guo, Nanoscale 2014, 6, 181

[22] H. Gu, X. Zhang, H. Wei, Y. Huang, S. Wei, Z. Guo, Chem. Soc. Rev. 2013, 42, 5907,

[23] K. Ding, H. Yang, Y. Cao, C. Zheng, S. B. Rapole, Z. Guo, Materials Chemistry and Physics 2013, 142(1), 403

[24] K. Ding, Y. Wang, L. Liu, Likun Liu, X. Zhang, Z. Guo, Journal of Applied Electrochemistry 2013, 43(6), 567-574,

[25] K. Ding, Y. Wang, H. Yang, C. Zheng, Y. Cao, H. Wei, Y. Wang, Z. Guo, Electrochemica Acta 2013, 100, 147.

[26] H. Wei, D. Ding, S. Wei, Z. Guo, Journal of Materials Chemistry A 2013, 1, 10805

[27] H. Wei, H. Gu, J. Guo, S. Wei, Z. Guo, ECS Journal of Solid State Science and Technology 2013, 2(10), M3008 
[28] H. Wei, H. Gu, J. Guo, S. Wei, Z. Guo, Journal of the Electrochemical Society 2013, 160(7), G3038

[29] H. Gu, J. Guo, S. Wei, Z. Guo, Journal of Applied Polymer Science 2013, 130(4), 2238

[30] J. Zhu, H. Gu, Z. Luo, N. Haldolaarachige, D.P. Young, S. Wei, Z. Guo 2012, Langmuir, 28(27), 10246

[31] J.Zhu, X. Zhang, N. Haldolaarachchige, Q. Wang, Z. Luo, J. Ryu, D.P. Young, S. Wei, Z. Guo, Journal of Materials Chemistry 2012, 22(11), 4996

[32] H. Gu, S.B. Rapole, Y. Huang, D. Cao, Z. Luo, S. Wei, Z. Guo, Journal of Materials Chemistry A 2013, 1, 2011.

[33] H. Gu, S. Tadakamalla, X. Zhang, Y. Huang, Y. Jiang, H.A. Colorado, Z. Luo, S. Wei, Z. Guo, Journal of Materials Chemistry C 2013, 1, 729.

[34] Y. Li, J. Zhu, S. Wei, J. Ryu, Q. Wang, L. Sun, Z. Guo, Macromolecular Chemistry and Physics 2011, 212, 2429.

[35] C. Velasco-Santos, A.L. Martinez-Hernandez, V. M. Castano, Composite Interfaces 2005, 11(8-9), 567

[36] A.G. Rinzlerl, J. Liu, H. Dai, P. Nikolaev, C.B. Huffman, F.J. Rodrıguez-Macıas, P.J. Boul, A.H. Lu, D. Heymann, D.T. Colbert, R.S. Lee, J.E. Fischer, A.M. Rao, P.C. Eklund, R.E. Smalley, Appl. Phys. A 1998, 67, 29

[37] Y. Feng, H. Zhang, Y. Hou, T. P. Mc Nicholas, D. Yuan, S. Yang, L. Ding, W. Feng, J. Liu, ACS Nano 2008, 2(8), 1634

[38] N. Dementev, S. Osswald, Y. Gogots, E. Borguet, J. Mater. Chem. 2009, 19, 7904 
[39] A. Yu, E. Bekyarova, M.E. Itkis, D. Fakhrutdinov, R. Webster, R.C. Haddon, J. Am.

Chem. Soc. 2006, 128(30), 9902

[40] B. Lin, U. Sundararaj, P. Potschke, DOI: 10.1002/mame.200500335

[41] T. Villmow, S. Pegel, P. Pötschke, G. Heinrich, Polymer, 2011, 52, 2276

[42] V. Datsyuk, M. Kalyva, K. Papagelis, J. Parthenios, D. Tasis, A. Siokou, I. Kallitsis, C. Galiotis, Carbon, 2008, 46, 833

[43] C. Ge, Y. Li, J-J Yin, Y. Liu, L. Wang, Y. Zhao, C. Chen, NPG Asia Materials, 2012, 4,32

[44] S.H. Tan, J.C. Goak, S.C. Hong, N. Lee, Carbon, 2008, 46(2), 245.

[45] T. Kashiwagi, E. Grulke, J. Hilding, K. Groth, R. Harris, K. Butler, J. Shields, S.

Kharchenko, J. Douglas, Polymer, 2004, 45, 4227

[46] T. D. Hapuarachchi, T. Peijs, Composites Part A: Applied Science and Manufacturing, 2010, 41,954

[47] M. Morreale, R. Scaffaro, A. Maio, F.P. La Mantia, Comp. Pt. A, 2008, 39(9), 1537

[48] R. Saito, G. Dresselhaus, M. S. Dresselhaus, Physical Properties of Carbon Nanotubes; London: Imperial College Press, 1998, 259 pp.

[49] Y. Wang, J. Wu, F. Wei, Carbon, 2003, 41, 2939

[50] S.H. Su, W.T. Chiang, C.C. Lin, M. Yokoyama, Physica E, 2008, 40, 2322

[51] L. Stobinski, B. Lesiak, L. Kover, J. Toth, S. Biniak, G. Trykowski, J. Judek, J. of Alloys and Compounds., 2010, 501, 77

[52] Saleh, T.A. App. Surf. Sc., 2011, 257, 7746 
[53] S.H. Tan, J.C. Goak, S.C. Hong, N. Lee, Carbon, 2008, 46, 245

[51] K. Chajara, C.H. Andersson, J. Lu, E. Widenkvist, H. Grennberg, New J. Chem. 2010, 34,2275

[52] Mazzocchia, C. V., Bestetti, M.; Acierno, D.; Tito, A. A process for the preparation of a catalyst, a catalyst obtained thereby, and its use in the production, Eur. Pat. 2213369 (A1), 2010

[53] N. G. Sahoo, S. Rana, J. W. Cho, L. Li, S. H. Chan, Progress in Polymer Science 2010, 35(7), 837

[54] Y.P. Sun, K. Fu, Y. Lin, W. Huang, Acc. Chem. Res., 2002, 35(12), 1096

[55] P.C. Ma, N. A. Siddiqui, G. Marom, J.-K Kim, Composites Part A: App. Sc. and Manufact. 2010, 41(10), 1345

[56] M. Morreale, R. Scaffaro, A. Maio, F.P. La Mantia, Comp. Pt. A, 2008, 39, 503

[57] R. Scaffaro, L. Botta, M. Ceraulo, F.P. La Mantia, J Appl Polym Sci, 2011, 122, 384.

[58] R. Scaffaro, L. Botta, M.C. Mistretta, F.P. La Mantia, Polymer Degradation and Stability, 2010, 95, 2547

[59] R. Scaffaro, M. Morreale, G. Lo Re, F.P. La Mantia, J Appl Polym Sci, 2009,114, 2855

[60] R. Scaffaro, L. Botta, F. P. La Mantia, Macromol Mater Eng. 2009, 294, 445

[61] Principles of polymer engineering, N.G. McCrum, C.P. Buckley, C.B. Bucknall, Oxford Science Publications 1997.

[62] A. Allaoui, S. Bai, H.M. Cheng, J.B Bai, Composites Science and Technology, 2002, 62,1993

[63] V. Datsyuk, M. Kalyva, K. Papagelis, J. Parthenios, D. Tasis, A. Siokou, I. Kallitsis, C. Galiotis, Carbon, 2008, 46, 833 
[64] N.I. Kovtyukhova, T.E. Mallouk, L. Pan, E.C. Dickey, J Am Chem Soc, 2003,125, 9761

[65] J Zhang, H Zou, Q Qing, Y Yang, Q Li, Z Liu, X Guo, Z Du, The Journal of Physical Chemistry B 2003, 107 (16), 3712

[66] F. Pourfayaz, Y. Mortazavi, A. Khodadadi, S. H. Jafari, S. Boroun, M. V. Naseh, Applied Surface Science, 2014, 295, 66

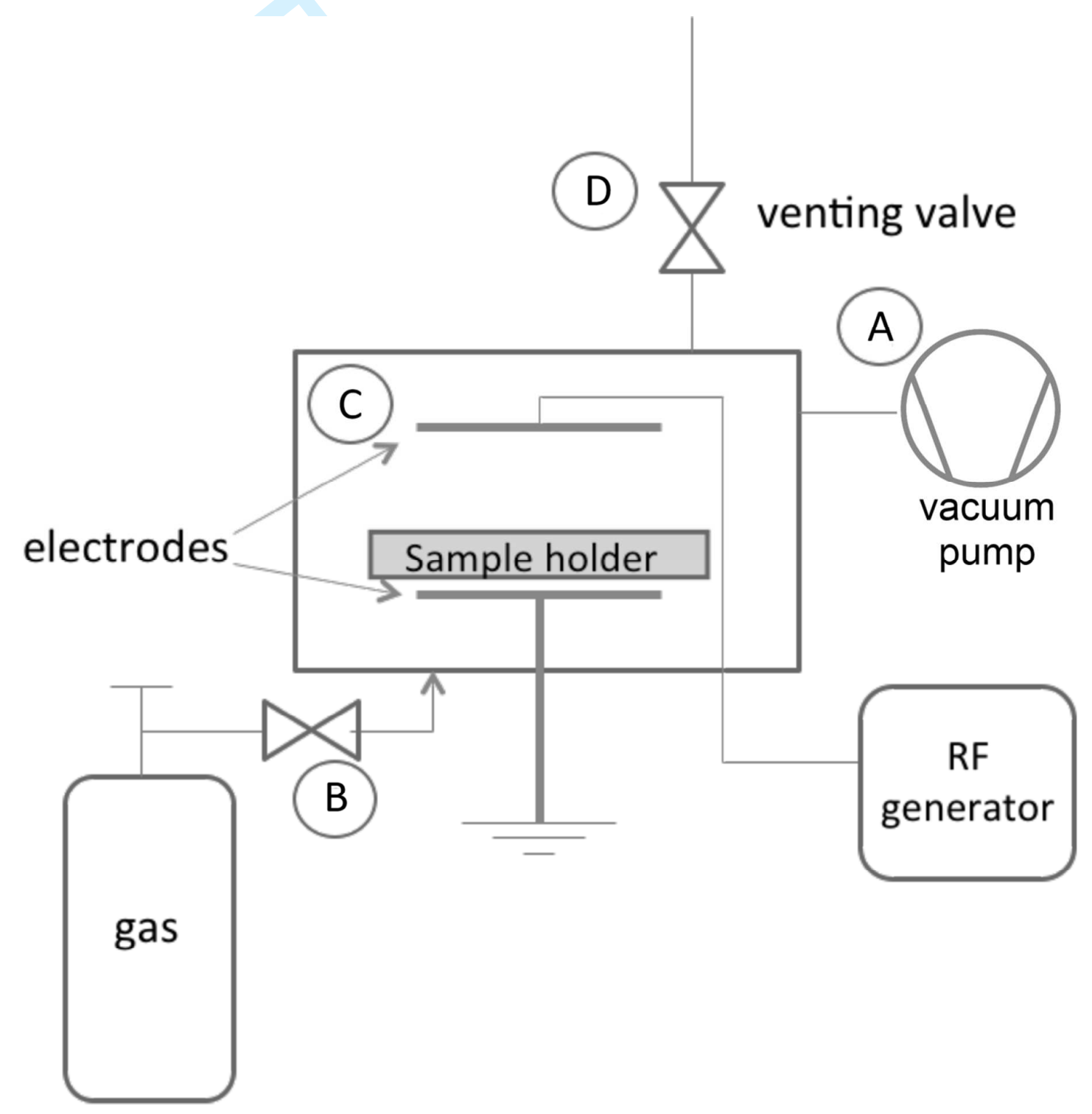

Scheme 1. RF-plasma reactor 

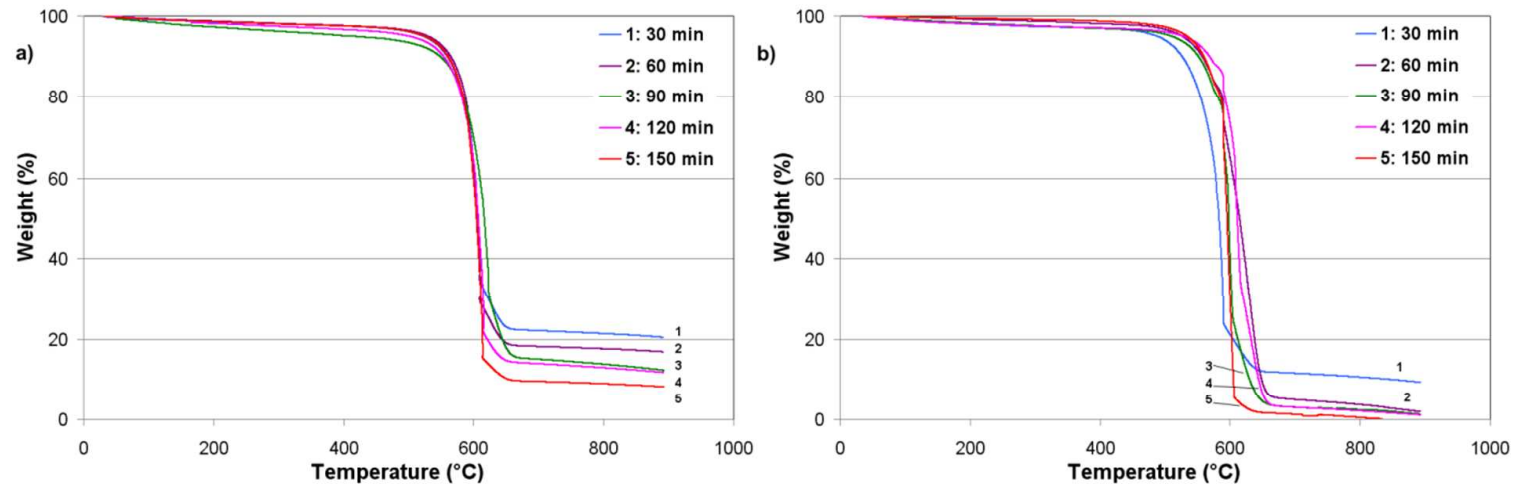

Figure 1. TGA (air atmosphere) of MWCNTs treated for different times with $45 \mathrm{wt} \%$ (a) and $70 \%$ (b) $\mathrm{H}_{2} \mathrm{SO}_{4}$ solution
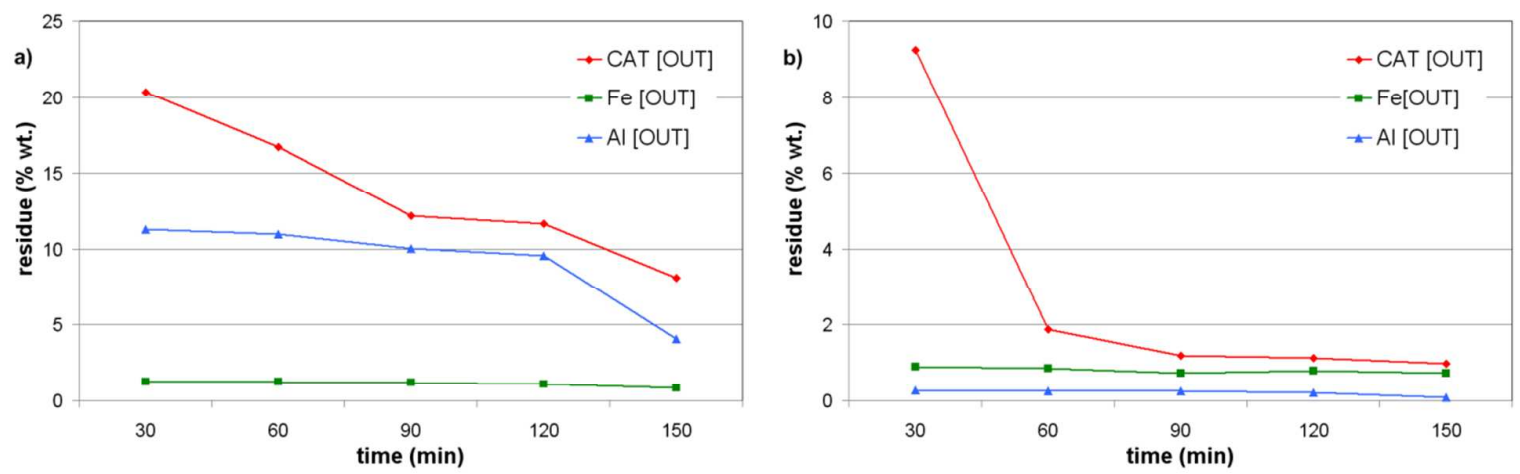

Figure 2. Residue weight as a function of purification time for MWCNTs treated with $45 \mathrm{wt} \%$ (a) and $70 \%$ (b) $\mathrm{H}_{2} \mathrm{SO}_{4}$ solution. 

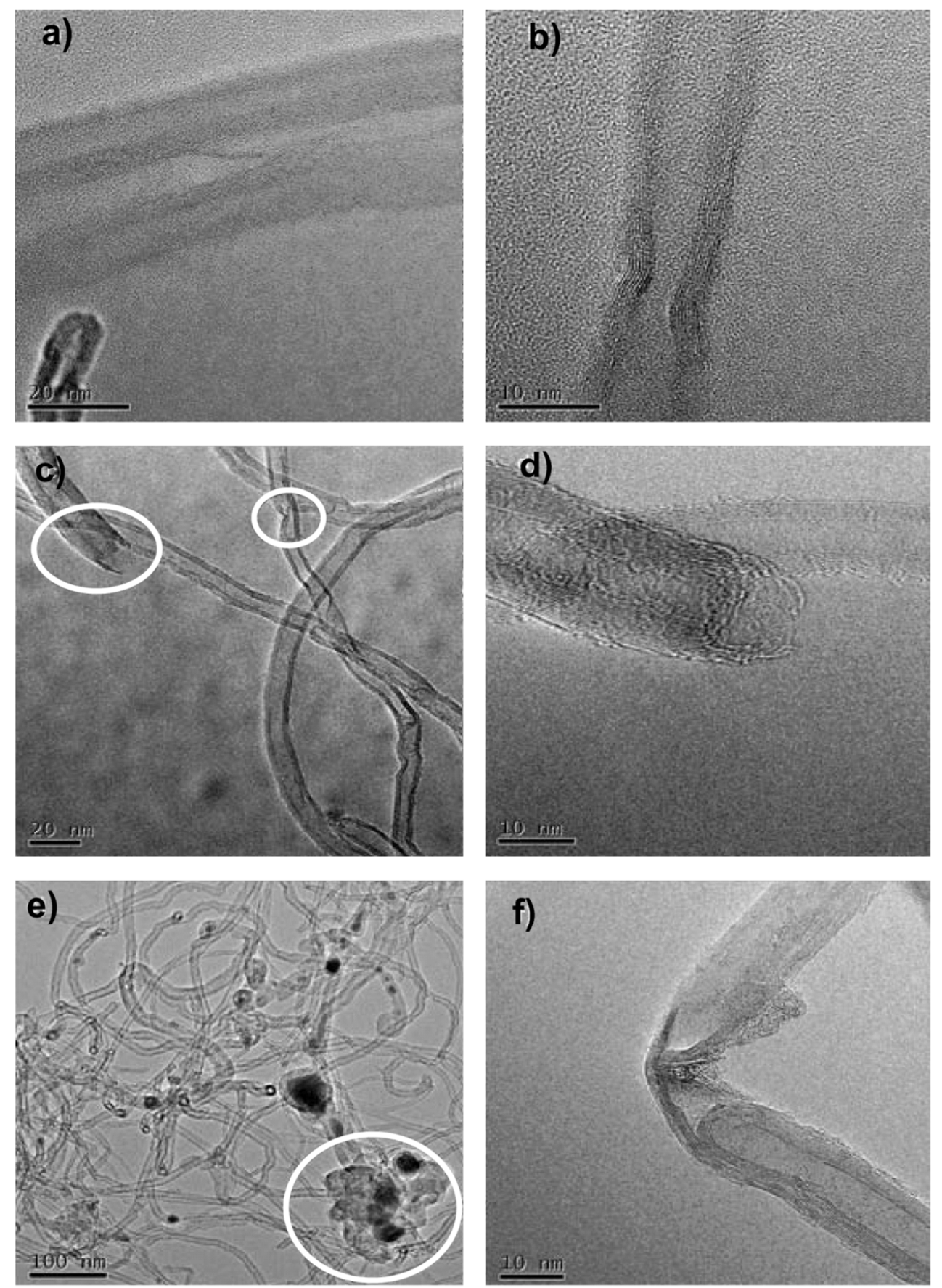

Figure 3. TEM: a)-b) as-prepared CNTs; c)-d) CNT 1, i.e. purified CNTs with $45 \mathrm{wt} \% \mathrm{H}_{2} \mathrm{SO}_{4}$ for 150 min.; e)-f) CNT 2, i.e. purified CNTs with $70 \mathrm{wt} \% \mathrm{H}_{2} \mathrm{SO}_{4}$ for $150 \mathrm{~min}$. 


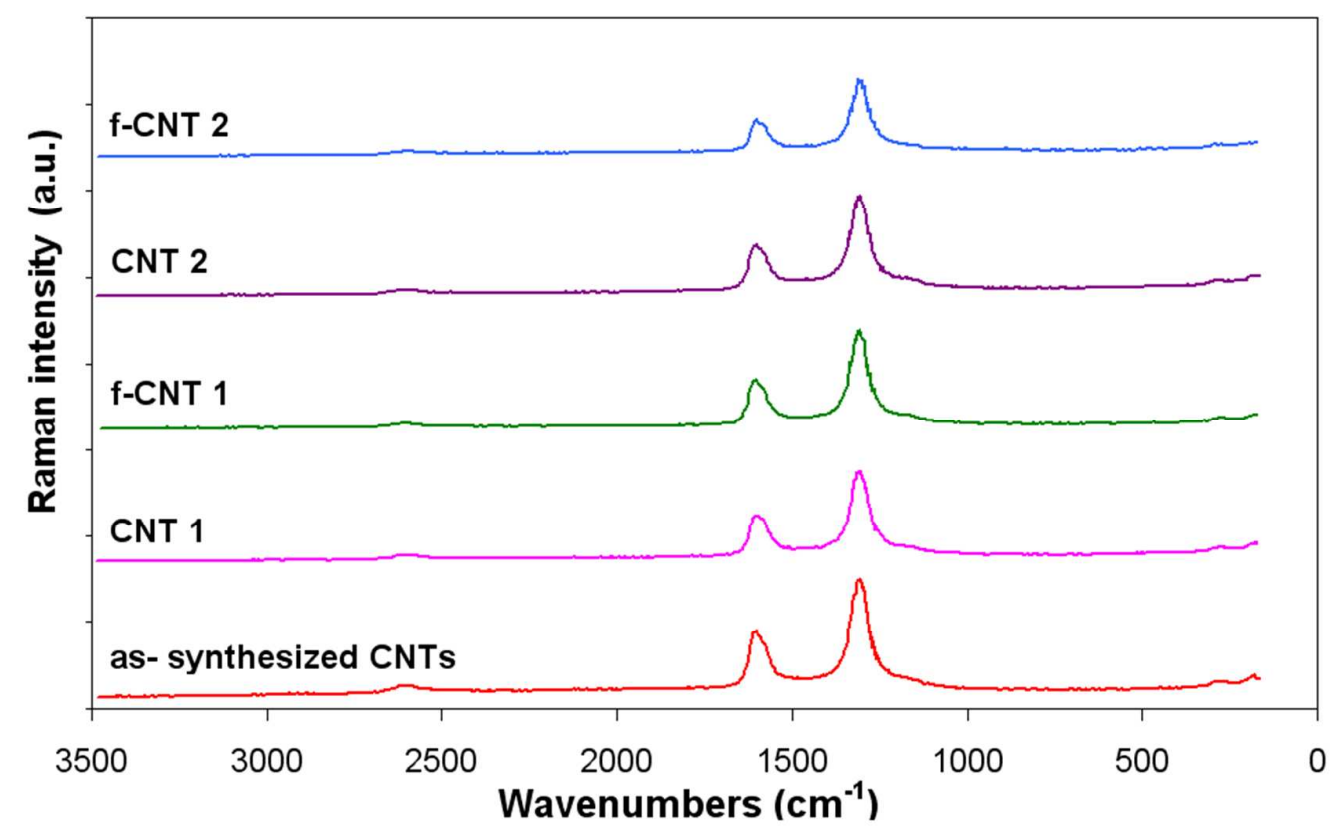

Figure 4. FT Raman spectra of as-synthesized nanotubes sample, CNT 1 (acid treatment: 45 wt $\% \mathrm{H}_{2} \mathrm{SO}_{4} 150 \mathrm{~min}$.), f-CNT 1 (acid treatment: $45 \mathrm{wt} \% \mathrm{H}_{2} \mathrm{SO}_{4} 150 \mathrm{~min}$, plus plasma treatment: $120 \mathrm{~W}, \mathrm{O}_{2}, 10 \mathrm{~min}$ ), CNT 2 (acid treatment: $70 \mathrm{wt} \% \mathrm{H}_{2} \mathrm{SO}_{4} 150 \mathrm{~min}$ ), f-CNT 2 (acid treatment: $70 \mathrm{wt} \% \mathrm{H}_{2} \mathrm{SO}_{4} 150 \mathrm{~min}$, plus plasma treatment: $120 \mathrm{~W}, \mathrm{O}_{2}, 10 \mathrm{~min}$ )

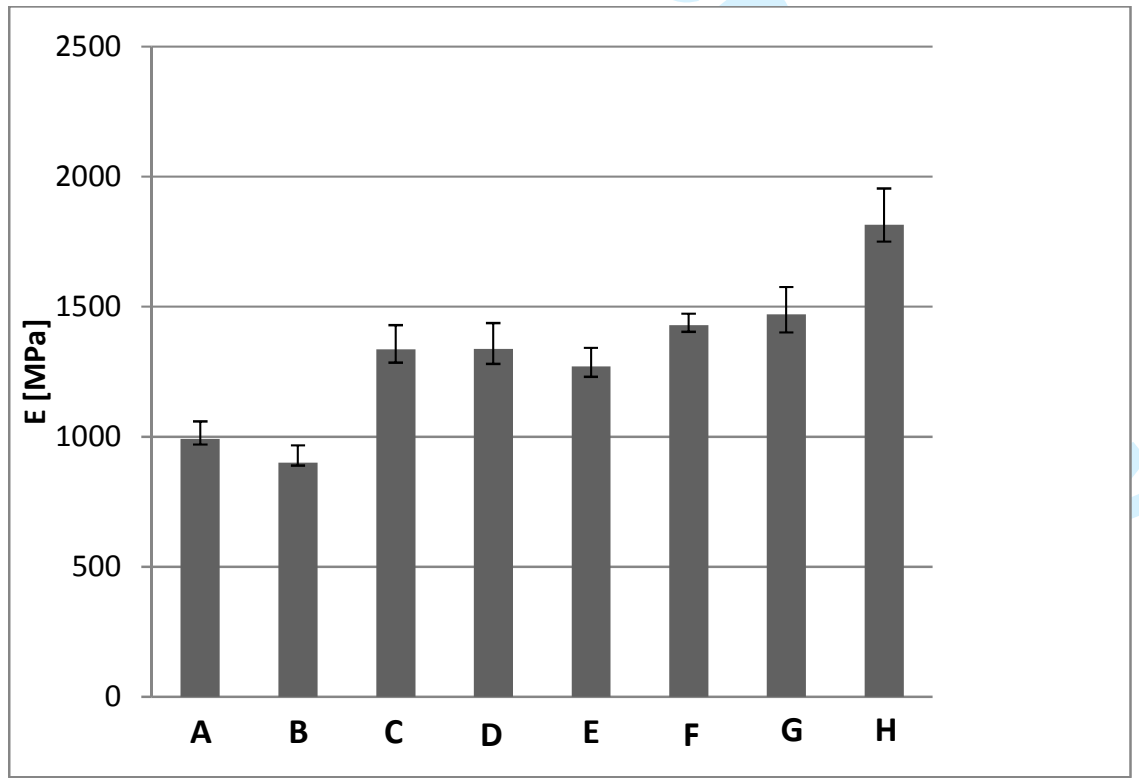

Figure 5. Data scattering for the elastic modulus of the 8 experimental runs. 


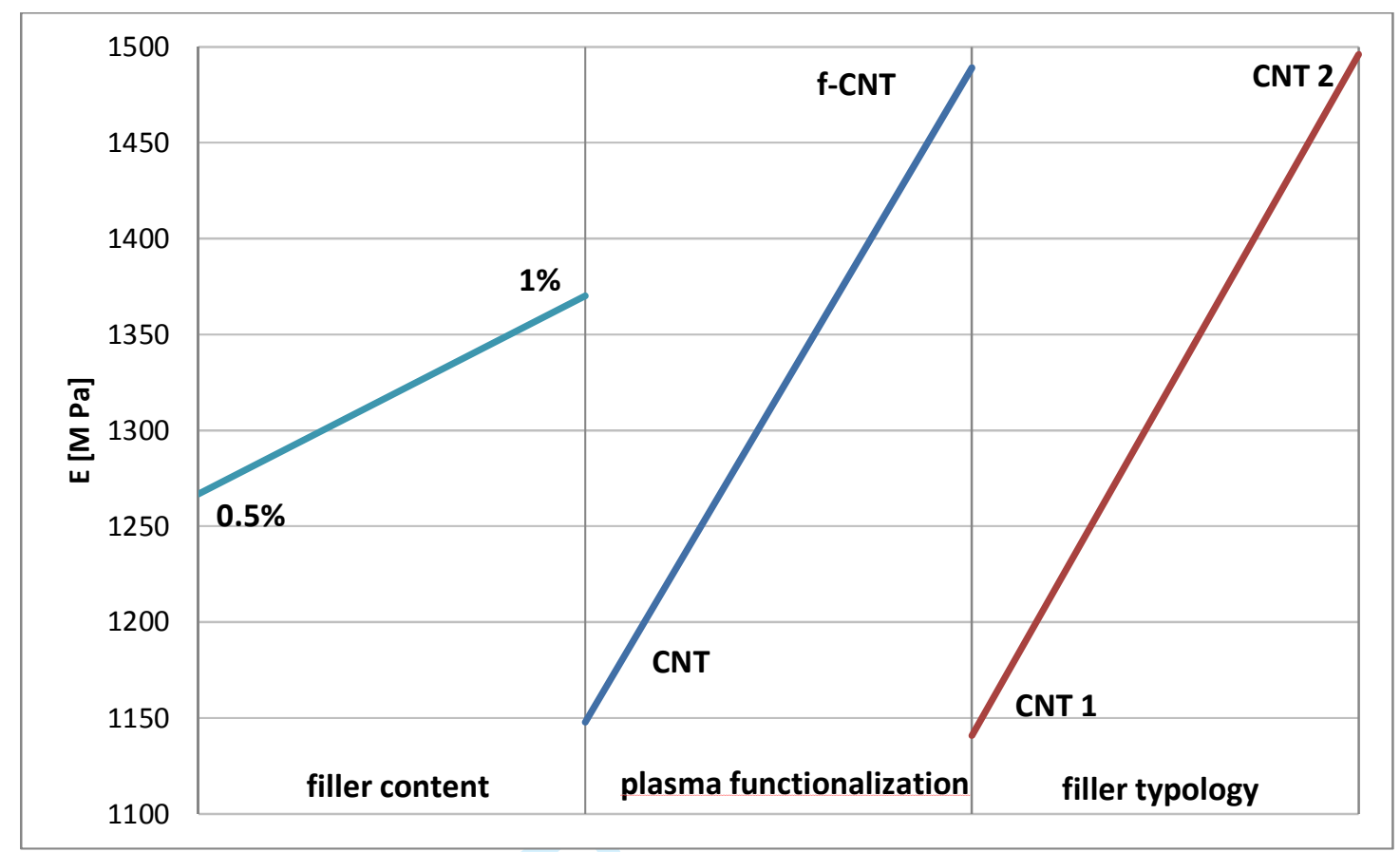

Figure 6. Main effect of filler content, functionalization and typology of CNTs on the tensile elastic modulus of PC/CNTs system.

a)

a) 1700

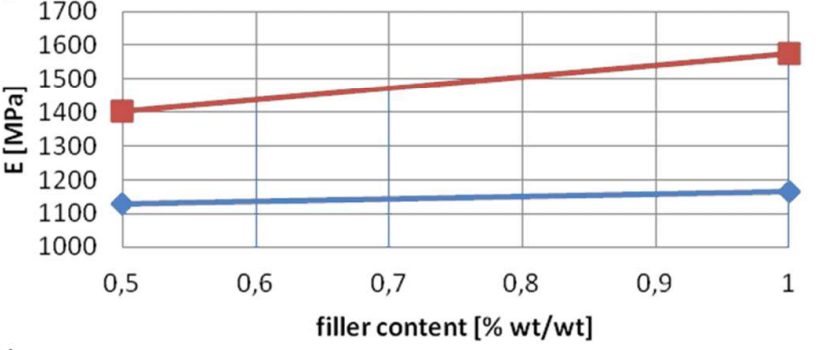

b)
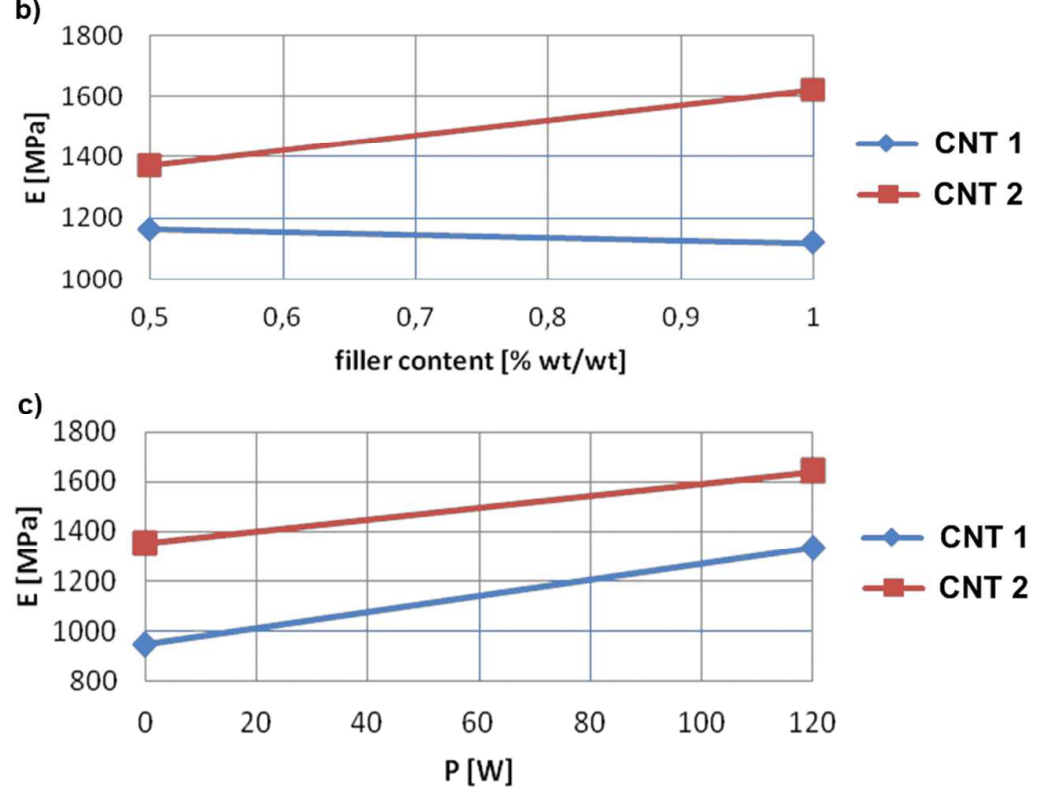

Figure 7. Map of the binary interactions for the system PC/CNT 

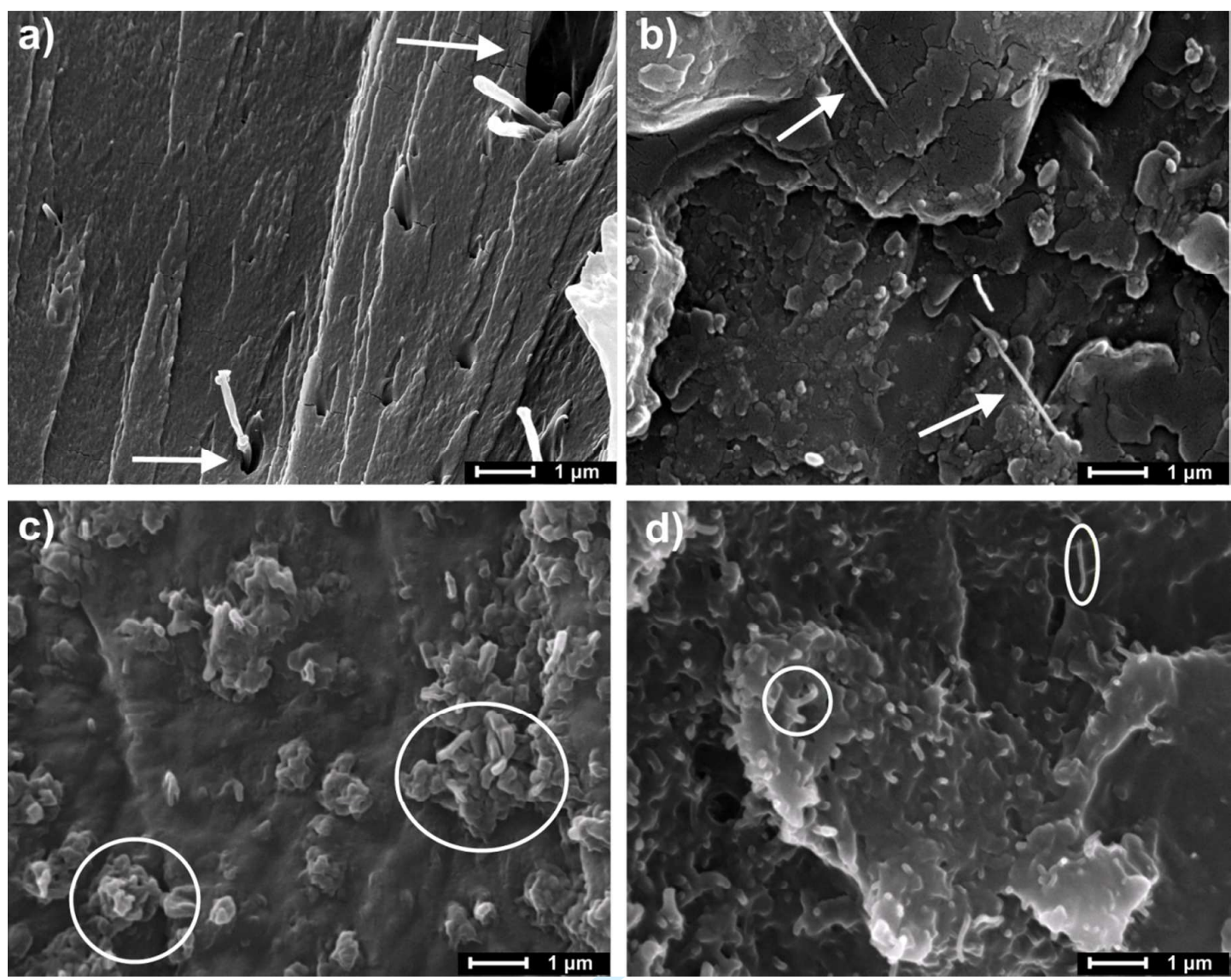

Figure 8. SEM micrographs of PC-based nanocomposites loaded with $1 \%$ of CNT 1, f-CNT 1, CNT 2 and f-CNT 2. 


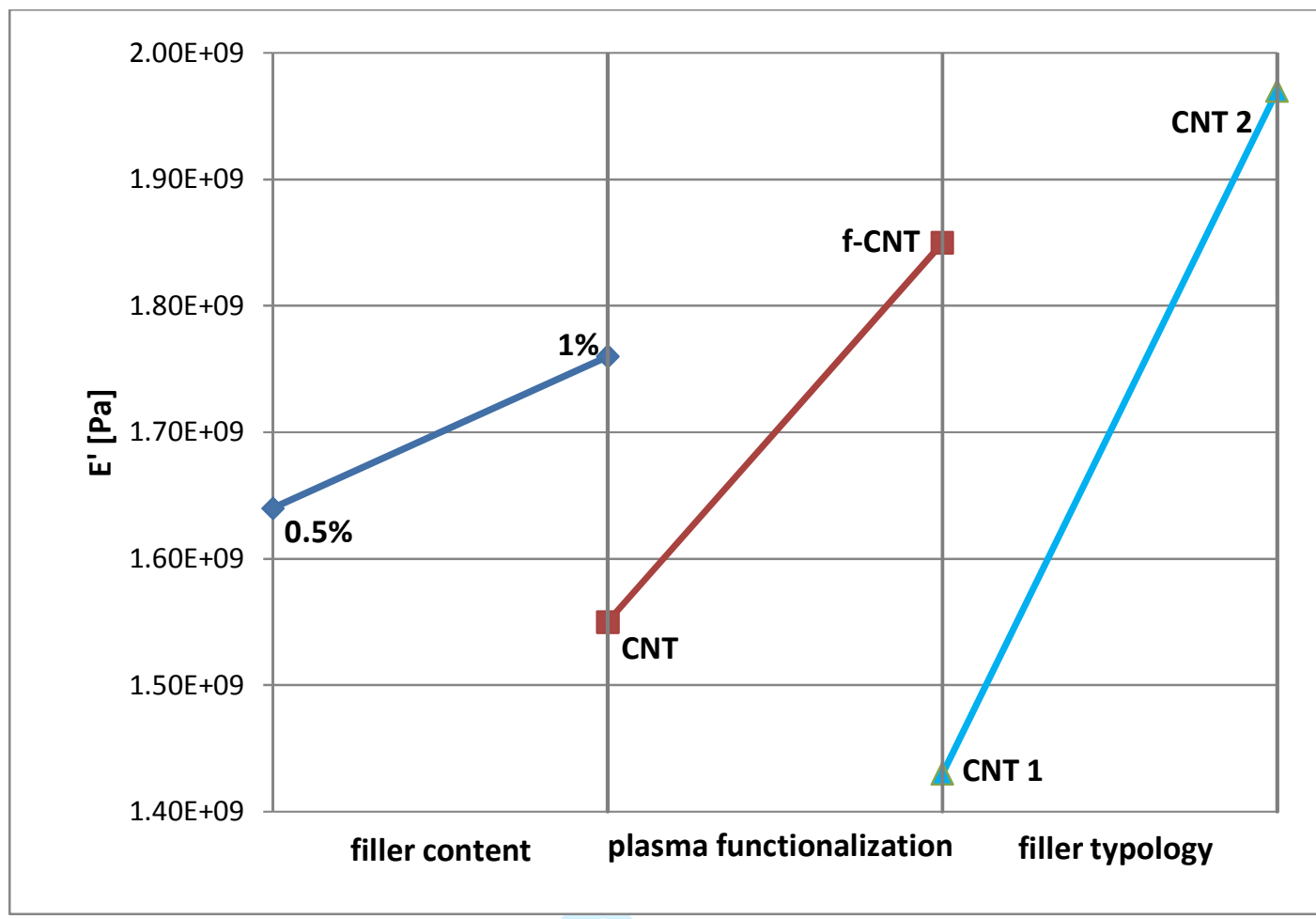

Figure 9. Main effect of content, typology and functionalization of the CNTs on the flexural storage modulus measured at $50^{\circ} \mathrm{C}$ of PC/CNTs nanocomposites.

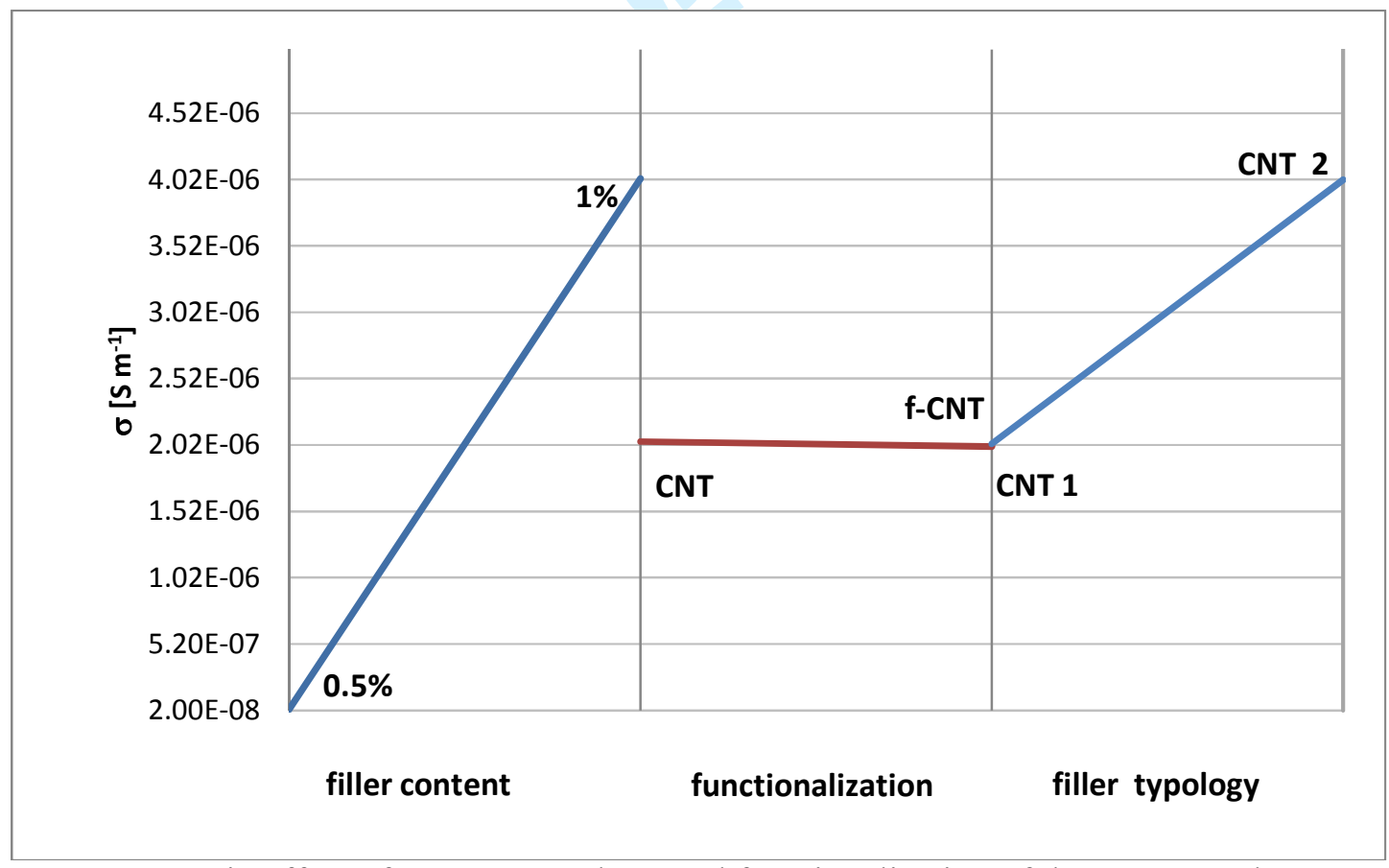

Figure 10. Main effect of content, typology and functionalization of the CNTs on the electrical conductivity of PC/CNTs nanocomposites. 

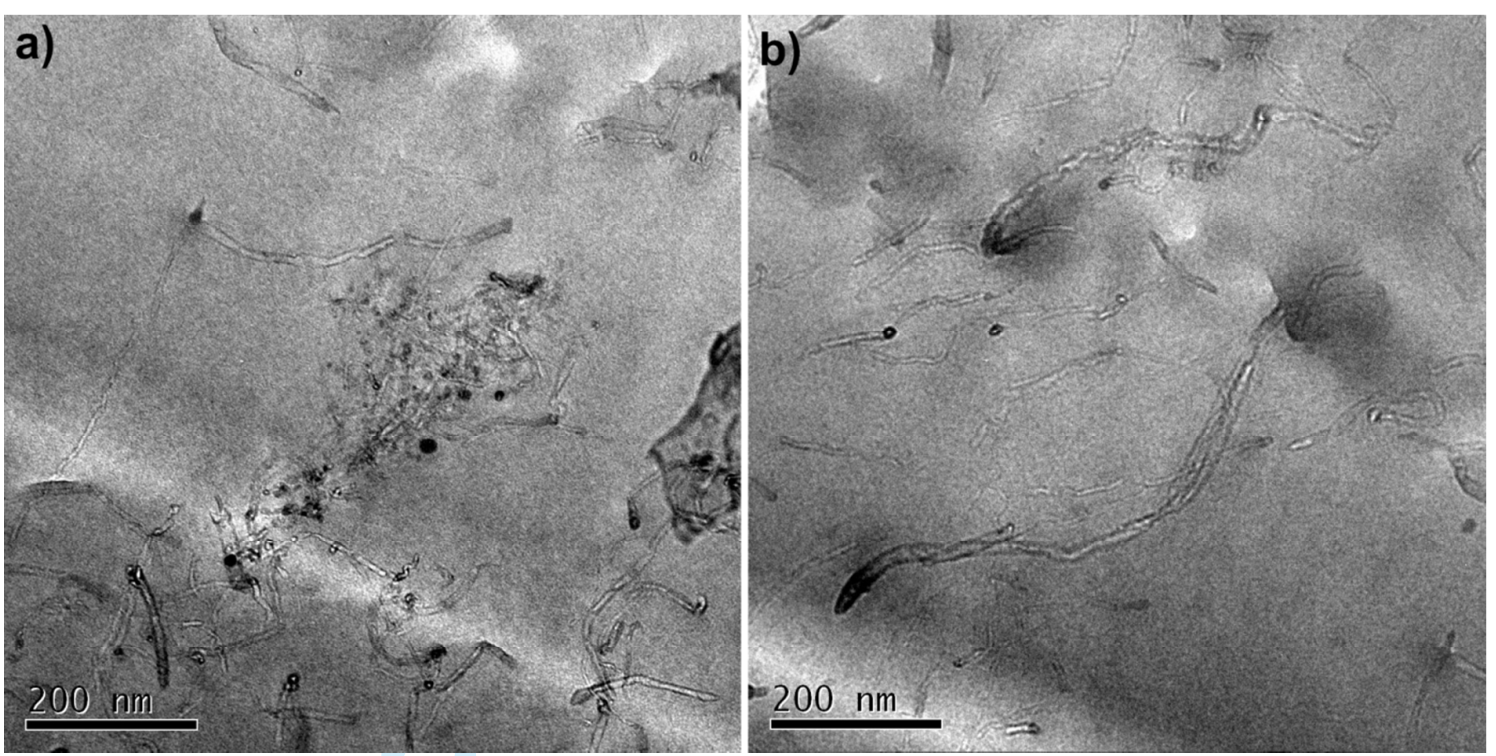

Figure 11. TEM micrographs of PC-based nanocomposites loaded with 1\% of CNT 2 (a) and f-CNT 2 (b)

Table 1. Main properties of the polycarbonate used in the present study

\begin{tabular}{|lcccccccc|}
\hline Sample & $\begin{array}{c}\text { Density } \\
{\left[\mathbf{g ~ c m}^{-3}\right]}\end{array}$ & $\begin{array}{c}\mathbf{T}_{\mathbf{g}} \\
{\left[{ }^{\circ} \mathbf{C}\right]}\end{array}$ & $\begin{array}{c}\mathbf{M}_{\mathbf{w}} \\
{[\mathbf{D a}]}\end{array}$ & $\begin{array}{c}\mathbf{M}_{\mathbf{n}} \\
{[\mathbf{D a}]}\end{array}$ & $\mathbf{M}_{\mathbf{w}} / \mathbf{M}_{\mathbf{n}}$ & $\begin{array}{c}\mathbf{E}^{\mathbf{a}} \\
{[\mathbf{M P a}]}\end{array}$ & $\begin{array}{c}\mathbf{E}^{{ }^{\mathbf{b}}} \\
{[\mathbf{G P a}]}\end{array}$ & $\begin{array}{c}\text { Conductivity } \\
{\left[\mathbf{S ~ m}^{\mathbf{- 1}}\right]}\end{array}$ \\
\hline $\begin{array}{l}\text { Lexan } \\
\text { HF }\end{array}$ & 1.13 & 148 & 36200 & 8700 & 4.16 & 736 & 1.5 & n.a. \\
1110 & & & & & & & & \\
\hline
\end{tabular}

${ }^{a}$ measured by tensile test according to ASTM D882; ${ }^{\text {) }}$ measured by flexural DMTA

following the same procedure adopted for the nanocomposites (see Experimental section)

Table 2. ICP/OES data and calculation of the degree of purity over time for CNTs purified adopting different acid concentrations.

\begin{tabular}{ccccccccc}
\hline \multicolumn{3}{c}{$\mathbf{4 5} \mathbf{w t} \% \mathbf{H}_{2} \mathbf{S O}_{4}$} & & \multicolumn{3}{c}{$\mathbf{7 0} \mathbf{w t} \% \mathbf{H}_{2} \mathbf{S O}_{4}$} \\
\hline $\begin{array}{c}\mathbf{t} \\
{[\mathbf{m i n}]}\end{array}$ & $\begin{array}{c}\mathbf{C A T} \text { out } \\
{[\mathbf{w t} \%]}\end{array}$ & $\begin{array}{c}\mathbf{F e}_{\text {out }} \\
{[\mathbf{w t} \%]}\end{array}$ & $\begin{array}{c}\mathbf{A l}_{\text {out }} \\
{[\mathbf{w t} \%]}\end{array}$ & $\begin{array}{c}\text { purity } \\
{[\mathbf{w t} \%]}\end{array}$ & $\begin{array}{c}\mathbf{C A T} \text { out } \\
{[\mathbf{w t} \%]}\end{array}$ & $\begin{array}{c}\mathbf{F e}_{\text {out }} \\
{[\mathbf{w t} \%]}\end{array}$ & $\begin{array}{c}\mathbf{A l}_{\text {out }} \\
{[\mathbf{w t} \%]}\end{array}$ & $\begin{array}{c}\text { purity } \\
{[\mathbf{w t} \%]}\end{array}$ \\
\hline 30 & 20.35 & 1.25 & 11.30 & 79.65 & 9.25 & 0.90 & 0.27 & 90.75 \\
60 & 16.72 & 1.23 & 11.00 & 83.28 & 1.88 & 0.85 & 0.25 & 98.11 \\
90 & 12.18 & 1.21 & 10.05 & 87.82 & 1.20 & 0.74 & 0.25 & 98.80 \\
120 & 11.64 & 1.1 & 9.55 & 88.36 & 1.13 & 0.80 & 0.20 & 98.90 \\
150 & 8.11 & 0.85 & 4.10 & 91.88 & 0.99 & 0.73 & 0.08 & 99.01
\end{tabular}


Table 3. $\mathrm{I}_{\mathrm{D}} / \mathrm{I}_{\mathrm{G}}$ ratio of as-synthesized nanotubes sample, CNT 1, f-CNT 1, CNT 2, f-CNT 2

$$
\text { Sample } \quad \mathbf{I}_{\mathbf{D}} / \mathbf{I}_{\mathbf{G}}
$$

As-synthesized

CNTs

CNT $1^{\text {a) }}$

f-CNT $1^{\text {b) }}$

$\mathrm{CNT} 2^{\mathrm{c})}$

f-CNT $2^{\text {d) }}$

a) acid treatment: $\left.45 \mathrm{wt} \% \mathrm{H}_{2} \mathrm{SO}_{4} 150 \mathrm{~min} ;{ }^{\mathrm{b}}\right)$ acid treatment ( $45 \mathrm{wt} \% \mathrm{H}_{2} \mathrm{SO}_{4} 150 \mathrm{~min}$ ) plus plasma treatment $\left(120 \mathrm{~W}, \mathrm{O}_{2}, 10 \mathrm{~min}\right) ;{ }^{\mathrm{c})}$ acid treatment $\left(70 \mathrm{wt} \% \mathrm{H}_{2} \mathrm{SO}_{4} 150 \mathrm{~min}\right) ;{ }^{\mathrm{d})}$ acid treatment $\left(70 \mathrm{wt} \% \mathrm{H}_{2} \mathrm{SO}_{4} 150 \mathrm{~min}\right)$ plus plasma treatment $\left(120 \mathrm{~W}, \mathrm{O}_{2}, 10 \mathrm{~min}\right)$

Table 4. Table of the contrasts for the interpretation of tensile elastic modulus in the PC/CNT system

\begin{tabular}{|c|c|c|c|c|c|c|c|c|c|}
\hline $\begin{array}{l}\text { Experimental } \\
\text { set conditions }\end{array}$ & $\begin{array}{c}1 \\
{[w t \%]}\end{array}$ & $\begin{array}{c}2 \\
\text { [plasma] }\end{array}$ & $\begin{array}{c}3 \\
\text { [typology] }\end{array}$ & $\begin{array}{c}1-2 \\
\text { interaction }\end{array}$ & $\begin{array}{c}1-3 \\
\text { interaction }\end{array}$ & $\begin{array}{c}2-3 \\
\text { interaction }\end{array}$ & $\begin{array}{c}\mathbf{E} \\
{[\mathrm{MPa}]}\end{array}$ & $\mathbf{E} / \mathbf{E}_{\mathbf{m}}$ & $\begin{array}{c}\text { variation } \\
\text { [\%] }\end{array}$ \\
\hline A & - & - & - & + & + & + & 991 & 1.346 & 34.65 \\
\hline B & + & - & - & - & - & + & 900 & 1.223 & 22.28 \\
\hline $\mathrm{C}$ & - & + & - & - & + & - & 1336 & 1.815 & 81.52 \\
\hline D & + & + & - & + & 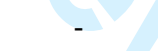 & - & 1336.5 & 1.816 & 81.59 \\
\hline $\mathrm{E}$ & - & - & + & + & - & - & 1271 & 1.727 & 72.69 \\
\hline $\mathrm{F}$ & + & - & + & - & + & - & 1429 & 1.942 & 94.16 \\
\hline G & - & + & + & - & - & + & 1469 & 1.996 & 99.59 \\
\hline $\mathrm{H}$ & + & + & + & + & + & + & 1815 & 2.466 & 146.6 \\
\hline Main effect & 103.38 & 341.38 & 355.13 & -16.6 & 148.63 & -49.38 & 1318.4 & 1.791 & 79.13 \\
\hline
\end{tabular}


Table 5. Analysis of the variance on tensile modulus.

\begin{tabular}{|c|c|c|c|c|}
\hline Sample & $\min$ & $\max$ & $d_{i}{ }^{2}$ & $d_{i}^{2} / 2 g$ \\
\hline $\mathbf{A}$ & 970 & 1037 & 4489 & 280.5625 \\
\hline B & 888 & 955 & 4489 & 280.5625 \\
\hline C & 1284 & 1377 & 8649 & 540.5625 \\
\hline D & 1280 & 1380 & 10000 & 625 \\
\hline $\mathbf{E}$ & 1231 & 1302 & 5041 & 315.0625 \\
\hline $\mathbf{F}$ & 1404 & 1447 & 1849 & 115.5625 \\
\hline $\mathbf{G}$ & 1400 & 1506 & 11236 & 702.25 \\
\hline $\mathbf{H}$ & 1750 & 1890 & 19600 & 1225 \\
\hline \multicolumn{3}{|c|}{$\mathrm{s}^{2}$} & \multicolumn{2}{|c|}{4084.5625} \\
\hline \multicolumn{3}{|c|}{$V(E)$} & \multicolumn{2}{|c|}{816.9125} \\
\hline \multicolumn{3}{|c|}{ SE } & \multicolumn{2}{|c|}{28.58168} \\
\hline \multicolumn{3}{|c|}{$\begin{array}{l}\text { Minimum threshold of statistical } \\
\text { significance }\end{array}$} & \multicolumn{2}{|c|}{65.73787} \\
\hline
\end{tabular}


A route combining both wet and dry chemistry methods allows achieving polymer-CNTs nanocomposites with the desired properties by optimizing synthesis, purification, plasma functionalization and concentration conditions of the nanofillers resorting to a statistical design. Plasma-processed CNTs were found to improve mechanical properties without affecting electrical properties of the composites.

A. Maio, L. Botta, A.C. Tito, L. Pellegrino, M. Daghetta, R. Scaffaro*

Statistical study of the influence of CNTs purification and plasma functionalization on the properties of Polycarbonate-CNTs nanocomposites

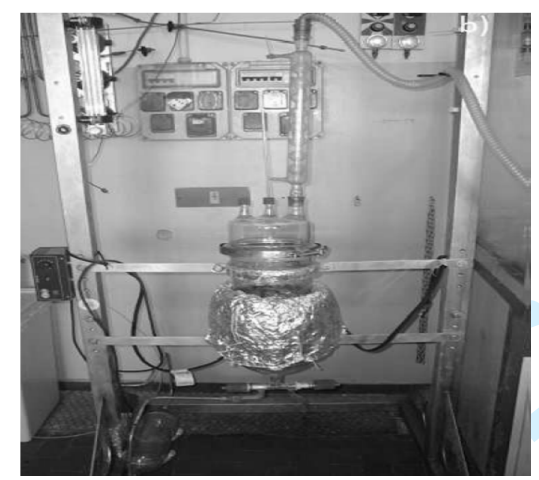

